\title{
Probing the flavor violating scalar top quark signal at the LHC
}

\author{
Genevieve Belanger ${ }^{a}$, Diptimoy Ghosh ${ }^{b}$, Rohini Godbole ${ }^{c}$, Monoranjan Guchait ${ }^{d}$ and \\ Dipan Sengupta ${ }^{d}$ \\ a) LAPTH, Univ. de Savoie, CNRS, \\ B.P.110, F-74941 Annecy-le-Vieux, France \\ b) INFN, Sezione di Roma, \\ Piazzale A. Moro 2, I-00185 Roma, Italy. \\ c) Center for High Energy Physics, Indian Institute of Science, \\ Bangalore, 560012, India. \\ d) Department of High Energy Physics, Tata Institute of Fundamental Research, \\ 1, Homi Bhabha Road, Mumbai 400 005, India.
}

\begin{abstract}
The Large Hadron Collider(LHC) has completed its run at $8 \mathrm{TeV}$ with the experiments ATLAS and CMS having collected about $25 \mathrm{fb}^{-1}$ of data each. Discovery of a light Higgs boson, coupled with lack of evidence for supersymmetry at the LHC so far, has motivated studies of supersymmetry in the context of naturalness with the principal focus being the third generation squarks. In this work, we analyze the prospects of the flavor violating decay mode $\tilde{\mathrm{t}}_{1} \rightarrow \mathrm{c} \chi_{1}^{0}$ at 8 and $13 \mathrm{TeV}$ center of mass energy at the LHC. This channel is also relevant in the dark matter context for the stop-coannihilation scenario, where the relic density depends on the mass difference between the lighter stop quark $\left(\tilde{t}_{1}\right)$ and the lightest neutralino $\left(\chi_{1}^{0}\right)$ states. This channel is extremely challenging to probe, specially for situations when the mass difference between the lighter stop quark and the lightest neutralino is small. Using certain kinematical properties of signal events we find that the level of backgrounds can be reduced substantially. We find that the prospect for this channel is limited due to the low production cross section for top squarks and limited luminosity at $8 \mathrm{TeV}$, but at the $13 \mathrm{TeV} \mathrm{LHC}$ with $100 \mathrm{fb}^{-1}$ luminosity, it is possible to probe top squarks with masses up to $\sim 450 \mathrm{GeV}$. We also discuss how the sensitivity could be significantly improved by tagging charm jets.
\end{abstract}




\section{Introduction}

At the end of the $8 \mathrm{TeV}$ run the Large Hadron Collider(LHC) has collected about $25 \mathrm{fb}^{-1}$ of data and produced the most defining observation since the onset of its operation in 2008. This observation is the finding of a new boson at $125 \mathrm{GeV}[1,2]$, which in all its glory seems to behave like the standard model(SM) Higgs boson. However, its true nature will be revealed only after precision measurements of its couplings and spin as well as their comparison with SM predictions. Interestingly, this discovery of the Higgs like boson has galvanized the beyond standard model(BSM) physics hunters in analyzing its impact on various new physics models including supersymmetry(SUSY). The implications of the $125 \mathrm{GeV}$ Higgs on SUSY have been well studied in the literature [3-34], and this is sure to continue in the coming days.

The early searches in SUSY, the most popular model among all the BSM options, have focussed, both phenomenologically [35-38] and experimentally [39,40], on the constrained minimal supersymmetric standard model(CMSSM)/minimal supergravity(mSUGRA) [41-46]. The initial searches probed mainly the gluino $(\tilde{g})$ and the squarks $(\tilde{q})$ of the first two generations. The current lower limits on the gluino and the first two generations squark masses in the framework of CMSSM stand at $\mathrm{m}_{\tilde{\mathrm{g}}} \gtrsim 1.5 \mathrm{TeV}$ for almost degenerate gluino and squarks and $\mathrm{m}_{\tilde{\mathrm{q}}}>1.4 \mathrm{TeV}$ for very high squark masses, [47-49].

The LHC constraints on the third generation squarks, stops $\left(\tilde{t}_{1,2}\right)$ and $\operatorname{sbottoms}\left(\tilde{b}_{1,2}\right)$ are weaker because of the lower production cross-section for a squark pair of a single flavor and are weakened even further when the mass difference between the squark and the neutralino is small. The negative results in the initial searches for the gluino and first two generations of squarks, together with the discovery of a 'light' Higgs boson have prompted consideration of natural SUSY, and hence a light third generation squark as an attractive framework for phenomenological studies.

Natural SUSY $[10,18,50-55]$ is inspired by the observation that the most relevant superparticles responsible for cancellation of quadratic divergences in the Higgs loop corrections are stop quarks. At the loop level the Higgs potential receives corrections from gauge and Yukawa interactions, the dominant part being the top-stop loop. The radiative corrections $\left(\delta m_{H_{u}}^{2}\right)$ are quadratically proportional to the third generation left and right soft masses along with the trilinear coupling $A_{t}$. Thus the trilinear couplings and the soft masses which control the third generation spectrum determine the level of fine tuning required to stabilize the Higgs mass in the theory. Another measure of 'naturalness' is also inspired by demanding that the level of fine tuning among different terms in the relation connecting $M_{Z}^{2}, \mu^{2}$ and the SUSY breaking scale is not high. These considerations thus imply light Higgsinos or light third generation squarks. The natural SUSY spectrum thus requires only a few particles below the $\mathrm{TeV}$ scale, the stops and the sbottom, the lighter charginos and neutralinos, and the gluino [10,54-56]. These natural SUSY scenarios have been studied in the literature for a wide range of phenomenological models and signatures. Furthermore, the anatomy of lighter third generation superparticles has been well dissected in the literature in terms of models and collider signatures [51,52,57-79].

Most of these studies, especially in the context of colliders have focused on the decay of lighter $\operatorname{stop}\left(\tilde{t}_{1}\right)$ and bottom $\left(\tilde{b}_{1}\right), \widetilde{\mathrm{t}}_{1} \rightarrow \mathrm{t} \chi_{1}^{0}$ and $\widetilde{\mathrm{b}}_{1} \rightarrow \mathrm{b} \chi_{1}^{0}$ respectively. It has been observed that the feasibility of these channels depend critically on the mass difference

$$
\Delta \mathrm{m}=\mathrm{m}_{\tilde{\mathrm{t}}_{1}\left(\widetilde{\mathrm{b}}_{1}\right)}-\mathrm{m}_{\chi_{1}^{0}},
$$

since it determines the hardness of the final state particles. A large value of $\Delta m$ leads to large jet momentum and a sizable missing transverse momentum $\left(\mathrm{p}_{\mathrm{T}}\right)$, both of which are imperative to 
suppress the SM backgrounds. Interestingly in the large $\Delta m$ scenario, the use of jet-substructure and top tagging is also an important tool to suppress backgrounds [80,81]. To alleviate the problem of low mass differences, shape analysis with various kinematic variables have also been considered [71].

As noted earlier the non-observation of a light sparticle in strongly interacting sector, i.e, a gluino and/or a squark of first two generation, in the sub-TeV regime along with the observation of a light Higgs have prompted ATLAS and CMS to perform dedicated searches for the third generations squarks. Note that, the limits on the masses of the squarks of first two generations using the generic SUSY searches are not applicable to the case of third generation squarks. The dedicated searches look for third generation squarks produced directly via QCD processes as well as those produced indirectly in gluino decays, in a plethora of final states coming from a variety of decay channels of $\tilde{t}_{1}$ assuming specific mass relationships among $\widetilde{\mathrm{g}}, \widetilde{\mathrm{t}}_{1}, \chi_{1}^{ \pm}$and the $\chi_{1}^{0}$, assumed to be the lightest SUSY particle(LSP). The principle decay channels studied are $\widetilde{t}_{1} \rightarrow \mathrm{t} \chi_{1}^{0}$ and $\widetilde{\mathrm{t}}_{1} \rightarrow \mathrm{b} \chi_{1}^{ \pm}$, with leptons and $\mathrm{b}$ jets in the final state from top quark decay. ATLAS searched for $\tilde{t}_{1}$ in the decay channel, $\tilde{\mathrm{t}}_{1} \rightarrow \mathrm{t} \chi_{1}^{0}$, from direct stop pair production using $8 \mathrm{TeV}$ LHC data with $13 \mathrm{fb}^{-1}$ luminosity and ruled out $m_{\tilde{t}_{1}}$ between $225 \mathrm{GeV}-575 \mathrm{GeV}$ for an LSP mass up to $175 \mathrm{GeV}$ [82]. ATLAS also probed $\tilde{t}_{1}$ in the channel $\widetilde{\mathrm{t}}_{1} \rightarrow \mathrm{b} \chi_{1}^{ \pm} \rightarrow \mathrm{b} \chi_{1}^{0} \mathrm{ff}^{\prime}$ assuming $\Delta \mathrm{m}=\mathrm{m}_{\chi_{1}^{ \pm}}-\mathrm{m}_{\chi_{1}^{0}}$ to be $5 \mathrm{GeV}$ and $20 \mathrm{GeV}$. For $\Delta \mathrm{m}=5 \mathrm{GeV}$ they ruled out stop masses of about $600 \mathrm{GeV}$ in a corridor of lightest neutralino mass [83]. With the use of the kinematic variable $\mathrm{M}_{\mathrm{T} 2}$, ATLAS also excluded $m_{\tilde{t}_{1}}$ in the range of $150 \mathrm{GeV}-450 \mathrm{GeV}$ for the channel $\widetilde{\mathrm{t}}_{1} \rightarrow \mathrm{b} \chi_{1}^{ \pm}$, where the chargino is nearly degenerate with the $\tilde{t}_{1}$ state [84]. Similarly, CMS searched for $\tilde{t}_{1}$ in the channels $\widetilde{\mathrm{t}}_{1} \rightarrow \mathrm{t} \chi_{1}^{0}$ and $\widetilde{\mathrm{t}}_{1} \rightarrow \mathrm{b} \chi_{1}^{ \pm}$, and excluded it with a mass between $160 \mathrm{GeV}-430 \mathrm{GeV}$ for a LSP mass up to $150 \mathrm{GeV}$ [85].

However, when the lighter stop becomes next to lightest SUSY particle(NLSP), the stop searches at colliders are quite different and become challenging. In this scenario, the dominant decay modes are via the flavor changing decays and the four body decay [86-88],

$$
\begin{aligned}
\tilde{t}_{1} & \rightarrow c \chi_{1}^{0} \\
& \rightarrow b f f^{\prime} \chi_{1}^{0} .
\end{aligned}
$$

Stop pair production followed by these decays leads to final states containing a heavy quark pair $c \bar{c}$ or $b \bar{b}$ respectively and are given by:

$$
\begin{gathered}
\mathrm{pp} \rightarrow \widetilde{\mathrm{t}_{1}} \widetilde{\mathrm{t}_{1}^{*}} \rightarrow \mathrm{c} \overline{\mathrm{c}}+2 \chi_{1}^{0} \rightarrow 2 \mathrm{jets}+\mathrm{p}_{\mathrm{T}}, \\
p p \rightarrow \widetilde{t_{1}} \widetilde{t_{1}^{*}} \rightarrow b \bar{b}+2 \chi_{1}^{0}+2 f f^{\prime} .
\end{gathered}
$$

The flavor violating decay mode yields precisely two jets and missing transverse momenta $\left(\not{ }_{T}\right)$ due to the presence of $\chi_{1}^{0}$. The relative decay rates into the above two channels are extremely sensitive to the model parameters $[88,89]$. The signal sensitivity for the four body decay channels has been studied for different parameters [90].

For very low values of $\Delta m$ (Eq. 1) it is rather difficult to obtain a reasonable signal sensitivity. In this case, the strategies have been to look at the mono-jet $+p_{T}^{\prime}[58,59]$ and mono-photon $+\not{ }_{T}$ final state [91,92]. The experimental limits on these channels come from reinterpretation of the monojet searches in ATLAS and CMS $[93,94]$. With the available data at $7 \mathrm{TeV}$, lighter stops of mass below about $200 \mathrm{GeV}$ are excluded for the above mentioned decay channels. Thus the limits on the mass of the lightest stop from these channels are rather weak. This is because the final state objects are soft due to the low value of $\Delta m$ leading to a lower acceptance of signal events. Therefore, it is a challenging task to probe these channels for very low $\Delta m$ cases. 
In this work we explore the possibility to find a signal for $\tilde{t_{1}}$ in the flavor violating decay, Eq. 4 , resulting in a di-jet $+p_{T}$ signature. Note that the flavor violating decay mode is also important in the dark matter context in the stop co-annihilation scenario [88]. The correct relic density abundance in this case crucially depends on $\Delta m$. In analyzing this signal we apply different types of kinematic selection cuts which are described in the following sections. For the case of flavour violating decay mode, the presence of c-quarks can be exploited by tagging c-jets. It is known that tagging c-jets is not easy because of the low mass of c quark and the low decay length of the charmed mesons. However, development of a strategy to tag the c-jets, even with a modest efciency will be helpful to suppress the SM backgrounds by an enormous amount and hence needs to be pursued.

The paper is organized as follows. In section 2 a brief description of the parameter space of interest is discussed. while in sections 3 and 4 we discuss our collider strategy and results respectively along with a discussion on the stop-coannihilation dark matter scenario. Finally we conclude in section 5 .

\section{Parameter Space}

\subsection{CMSSM and PMSSM}

We simulate the signal (Eq.4) for the parameter space of our interest in the context of both CMSSM and the phenomenological MSSM (pMSSM) with 19 parameters [95]. The CMSSM has been the most popular model of SUSY breaking in the context of collider phenomenology and experimental searches over the last two decades. This is primarily driven by the economy of the model which requires 4 parameters and a sign, as compared to the cornucopia of over 100 parameters in the MSSM. These parameters defined at the GUT scale, include the universal scalar mass $\left(\mathrm{m}_{0}\right)$, the universal fermion mass $\left(m_{1 / 2}\right)$ and the universal trilinear coupling $\mathrm{A}_{0}$, along with $\tan \beta$, the ratio of vacuum expectation values(VEV) of the two Higgs doublets and the sign of $\mu$, the Higgsino mass parameter, at the weak scale. The sparticle spectrum at the electroweak scale is obtained by renormalization group running from the GUT scale to the electroweak scale.

It is a well known fact the Higgs mass receives a substantial quantum correction resulting an enhancement of its mass from its tree level values which is bounded by mass of $Z$ boson, viz, $M_{H}^{2} \leq$ $\mathrm{M}_{\mathrm{Z}}^{2} \cos ^{2} 2 \beta$. In order to accommodate the Higgs mass of $125 \mathrm{GeV}$ in the CMSSM framework one necessarily requires stops in the multi-TeV regime or lighter stops with maximal mixing scenarios. Loop corrections can increase the tree level Higgs mass up to $\sim 140 \mathrm{GeV}$, due to stop-top loops and a large value of the triline ar coupling $A_{t}[3,4]$. However it has also been noted that such large trilinear coupling introduces a significant amount of fine tuning in the theory. On the other hand a large $\mathrm{A}_{0}$ results in a large splitting in the stop mass matrix. This means that lighter stops are accessible at LHC energies even in CMSSM [4]. Hence it is worth investigating, in the CMSSM, the available parameter space, which provides $\mathrm{m}_{\mathrm{H}} \simeq 125 \mathrm{GeV}$ and where we have imposed various experimental and theoretical constraints as described below.

\subsection{Constraining CMSSM}

With this goal, we perform a numerical scan of the relevant part of the CMSSM parameter space, varying the range of parameters such as,

$$
m_{0}:[0-3 \mathrm{TeV}], m_{1 / 2}:[0-1 \mathrm{TeV}], A_{0}:[-2-10 \mathrm{TeV}],
$$


and setting the top quark mass to be $172.9 \mathrm{GeV}$. We generate $5 \times 10^{5}$ random parameter points for a fixed value of $\tan \beta$. We fix the sign of the Higgsino mass parameter $\mu$ to be positive. We use the SusPect [96] spectrum generator to generate the masses of the supersymmetric particles for a fixed set of input parameters along with SuperIso [97] for the calculations of the branching ratios of rare B-meson decays.

We refer to a point in the CMSSM parameter space to be allowed if it survives the following constraints:

(i) The lightest Higgs boson mass $M_{H}$ falls in the window $122.5 \mathrm{GeV}<M_{H}<129.5 \mathrm{GeV}$. Note that, owing to the small difference in the central values given by the ATLAS and CMS collaborations [1,2], in order to be conservative, we used the number $126 \pm 2 \mathrm{GeV}$ as the experimental value. In addition, we added a $1.5 \mathrm{GeV}$ theoretical uncertainty following [29].

(ii) The branching ratio $(\mathscr{B})$ of the radiative decay $B \rightarrow X_{s} \gamma$ satisfies the following 95\% C.L. bound [98],

$$
2.6 \times 10^{-4}<\mathscr{B}\left(B \rightarrow X_{s} \gamma\right)<4.5 \times 10^{-4} .
$$

The HFAG Average and the SM predictions for this branching ratio are given by $[3.55 \pm 0.24 \pm$ $0.09] \times 10^{-4}$ [99] and $[3.15 \pm 0.23] \times 10^{-4}$ [100] respectively. We obtained the above $95 \%$ C.L. bound after including the intrinsic MSSM uncertainty of $0.15 \times 10^{-4}$, following the prescription given in [101].

(iii) The branching ratio of $B_{s} \rightarrow \mu^{+} \mu^{-}[102,103]$ lies in the $95 \%$ C.L. allowed range

$$
1.3 \times 10^{-9}<\mathscr{B}\left(B_{s} \rightarrow \mu^{+} \mu^{-}\right)<4.5 \times 10^{-9} .
$$

In order to get the above range we used the $\mathrm{CMS}+\mathrm{LHCb}$ average and $\mathrm{SM}$ predictions to be $[2.9 \pm$ $0.7] \times 10^{-9}[102,103]$ and $[3.56 \pm 0.18] \times 10^{-9}[100]$ respectively.

Figures $1 \& 2$ show the results of our numerical study.

If a point is allowed by all of the three constraints above then the point is plotted in magenta (dark grey). A point is green (pale grey) if it satisfies only the constraint (i) but none of the other two. Note that all the points (except the red (black) points in the $m_{0}-m_{1 / 2}$ plane) have been checked to satisfy the requirements of electroweak symmetry breaking, electric and color neutral LSP, the LEP lower bounds on the masses and other theoretical consistencies e.g., absence of tachyonic states and so on. It is worth mentioning here that the impact of the measured higgs mass on the parameter space is rather strong; the region with low values of $m_{0}$ and $m_{1 / 2}$ is completely ruled out by this single measurement (i). The absence of green points for $\tan \beta=10$ shows that the bounds from (ii) and (iii) above are not strong enough to rule out any point which is not already disallowed by the Higgs mass. This situation changes gradually as we go towards larger $\tan \beta$ values as can be seen from Fig. 2. In Fig. 2 there are many (green) points which are allowed by the Higgs mass but disfavored by the flavor physics data, in particular (iii). This happens because of the strong dependence of the branching ratios of $B \rightarrow X_{s} \gamma$ and $B_{s} \rightarrow \mu^{+} \mu^{-}$on $\tan \beta$. In fact, the dominant Higgs contribution to these branching ratios are proportional to $(\tan \beta)^{2}$ (for $\mathscr{B}\left(B \rightarrow X_{s} \gamma\right)$ ) and $(\tan \beta)^{6}$ (for $\mathscr{B}\left(B_{s} \rightarrow \mu^{+} \mu^{-}\right)$). This $(\tan \beta)^{6}$ dependence removes all the points with $m_{0}<3000$ $\mathrm{GeV}$ and $m_{1 / 2}<1000 \mathrm{GeV}$ for $\tan \beta=50$, hence we do not show them here. We would also like to point out in passing that the dominant Higgs mediated diagrams mentioned above are also proportional to the stop tri-linear coupling $A_{t}$. Thus the constraints from (ii) and (iii) can be relaxed for small values of $A_{t}$. On the other hand, the measured value of the Higgs mass prefers a large value of $A_{t}$. Hence, there is a complementarity between the bounds coming from the Higgs mass and those coming from the flavor physics data. 
In both Fig. 1 and Fig. 2 we also show (in the second row) the allowed values of $m_{\tilde{t}_{1}}$ for a wide range of $m_{1 / 2}$ and $A_{0}$ values. In spite of the constraints discussed above, it is quite clear from these figures that even in the CMSSM, there exists regions in the parameter space where a light stop below a TeV mass scale is allowed.

\subsection{Stop decay and benchmarks}

While it is certainly interesting to find a large region of parameter space pertaining to lighter stops and allowed by the Higgs mass constraint, the specific mass relations in CMSSM ties our hands to a large extent. In the context of natural SUSY it is enough to consider only the third generation squarks (stops and sbottoms), the third generation trilinear couplings along with charginos and neutralinos. The rest of the spectrum is mostly unimportant and can be decoupled from this set. This rather simplified approach, in the framework of the phenomenological MSSM (pMSSM) brings out the relevant physics with the minimal number of input parameters.

The parameter space of our interest is guided by the region where the flavor violating decay $\widetilde{\mathrm{t}}_{1} \rightarrow \mathrm{c} \chi_{1}^{0}$, is kinematically dominant for relatively small mass differences corresponding to Eq. 1 . The decay width is given by $[86,87]$,

$$
\Gamma=\frac{1}{2} \alpha|\varepsilon|^{2} \mathrm{~m}_{\tilde{\mathfrak{t}}_{1}}\left[1-\frac{\mathrm{m}_{\chi_{1}^{0}}^{2}}{\mathrm{~m}_{\tilde{\mathfrak{t}}_{1}}^{2}}\right]^{2},
$$

where the loop factor $\varepsilon$ is directly proportional to $\mathrm{A}_{\mathrm{b}}^{2}$ and $\tan ^{2} \beta$ with $\alpha$ being the strong coupling constant ${ }^{1}$.

A competing decay mode to the two body is the four body decay $\left(\tilde{t}_{1} \rightarrow b \chi_{1}^{0} f f^{\prime}\right)[86,87,89]$, dominantly via an off shell chargino into fermions. The two body decay mode, which is quadratically dependent on $\tan \beta$ and $\mathrm{A}_{\mathrm{b}}$ dominates over the four body decay for moderate to high $\tan \beta$. In the large $\tan \beta$ scenario, the two body decay mode dominates over the four body decay mode, for similar mass differences as compared to lower $\tan \beta$.

We choose two benchmark points in the CMSSM and four points in the 19 parameter pMSSM for our collider analysis. The representative points are shown in Tables 1 and 2, indicating the relevant branching ratios and other sparticle masses. All points in CMSSM (P1,P2) and three points in $\operatorname{pMSSM}(\mathrm{P} 3, \mathrm{P} 4, \mathrm{P} 5)$ are chosen to have $\tan \beta=10$. We choose one additional benchmark point with $\tan \beta=30$ in $\mathrm{pMSSM}(\mathrm{P} 6)$.

\begin{tabular}{|l|c|c|c|c|c|c|c|c|c|c|}
\hline & $\tan \beta$ & $m_{0}$ & $m_{1 / 2}$ & $A_{0}$ & $m_{H}$ & $m_{\tilde{t}_{1}}$ & $m_{\chi_{1}^{0}}$ & $\Delta m$ & $\begin{array}{c}\operatorname{Br}\left(\tilde{t}_{1} \rightarrow c \chi_{1}^{0}\right) \\
\%\end{array}$ & $\begin{array}{c}\operatorname{Br}\left(\tilde{t}_{1} \rightarrow b f f^{\prime} \chi_{1}^{0}\right) \\
\%\end{array}$ \\
\hline P1 & 10 & 1848 & 457.6 & -4069 & 126.0 & 241 & 198 & 43 & 74 & 25 \\
\hline P2 & 10 & 2589 & 695 & -5849 & 126 & 331 & 306 & 25 & 97 & 2 \\
\hline
\end{tabular}

Table 1: Masses and branching ratios of some of the particles in the CMSSM scenario. All energy units in GeV. $\operatorname{sgn}(\mu)$ is set to be positive.

For the pMSSM benchmarks in Table 2, we set the first two generation of squarks and all slepton generations to $5 \mathrm{TeV}$ and the gluino to $1.5 \mathrm{TeV}$ as they are irrelevant for our study. The parameter $M_{2}$ is set to $900 \mathrm{GeV}$. The trilinear couplings with the exception of $\mathrm{A}_{t}$ are all set to zero. The

\footnotetext{
${ }^{1}$ For an exact one-loop calculation of this decay width see [87].
} 

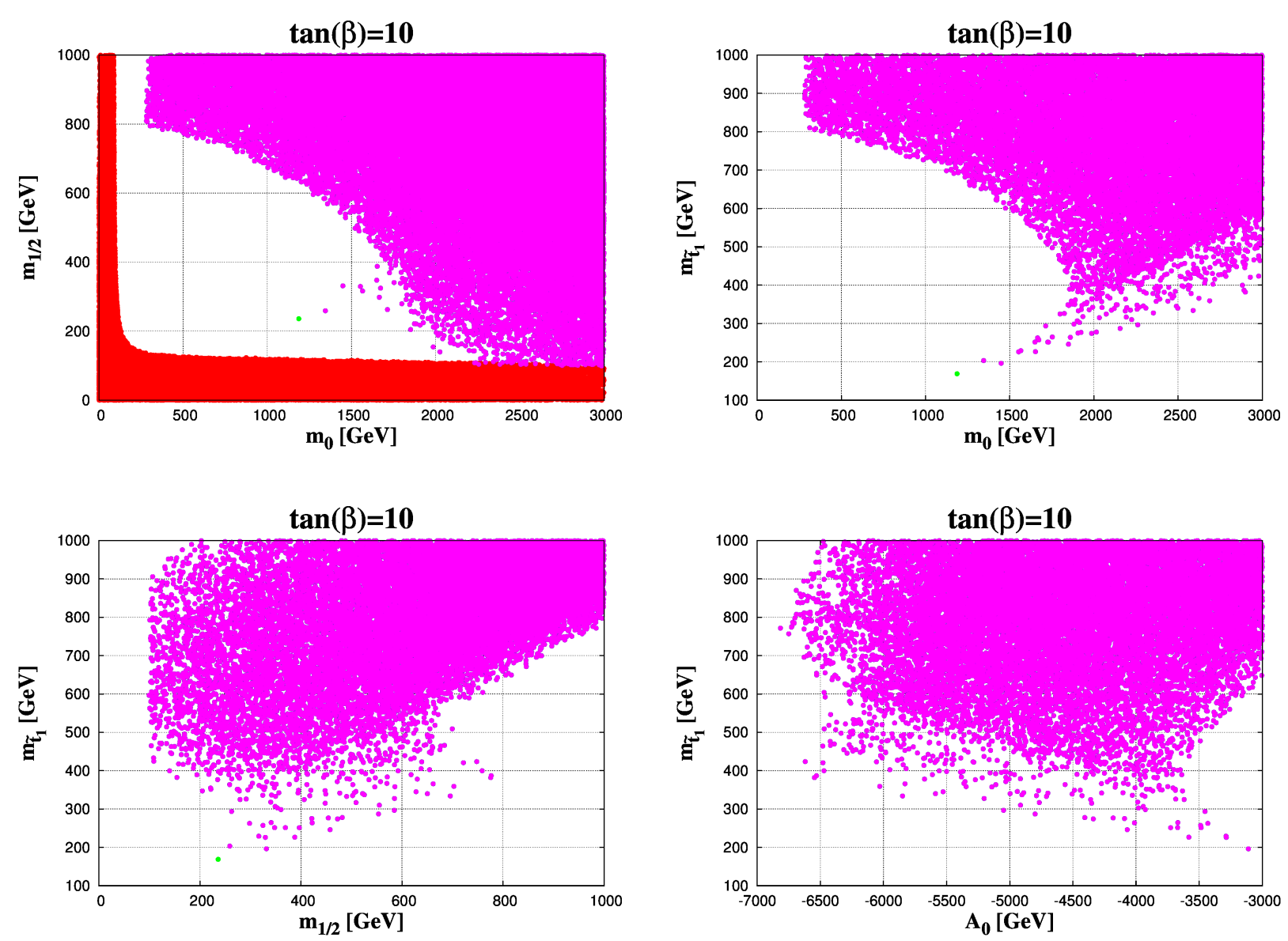

Figure 1: The allowed parameter points in the $m_{0}-m_{1 / 2}$ (top left panel), $m_{0}-m_{\tilde{t}_{1}}$ (top right panel), $m_{1 / 2}-m_{\tilde{t}_{1}}$ (middle left panel), $A_{0}-m_{\tilde{t}_{1}}$ (middle right panel), in the CMSSM scenario for $\tan \beta=10$. The red (black) region in the top left panel corresponds to points that are theoretically inconsistent. All the three constraints mentioned in the text are satisfied for the magenta (dark grey) points, whereas for the green (pale grey) points only the Higgs mass constraint is satisfied but the B-physics constraints are not.

pseudoscalar mass $m_{A}$ is set to $500 \mathrm{GeV}$. All the benchmark points have been checked against the constraints mentioned in Sec. 2.2.

\section{Signal and background}

We simulate the collider signatures of the stop pair production (Eq .4) for the benchmark points as shown in Tables 1 and 2 in the dijet $+p_{T}^{\prime}$ scenario and the corresponding SM background at the LHC. As pointed earlier, the final state objects like jets and $p_{T}$ are expected to be soft because of low $\Delta m$ making it difficult to obtain a reasonable acceptance after cuts necessary to suppress the $\mathrm{SM}$ backgrounds. The signal cross section is rather small at $8 \mathrm{TeV}$ and falls to $\sim 50 \mathrm{fb}$ for a stop mass of $500 \mathrm{GeV}$. Most of the search strategies proposed have taken recourse to monojet $+\not p_{T}$ or monophoton $+\not p_{T}$ searches, where a hard QCD jet is used along with a large $p_{T}$ [59]. It was demonstrated in [59] that with this strategy it is possible to use this channel for stop discovery, for stop masses up to $\sim 300 \mathrm{GeV}$ at $14 \mathrm{TeV}$ LHC, with $100 \mathrm{fb}^{-1}$ luminosity. 

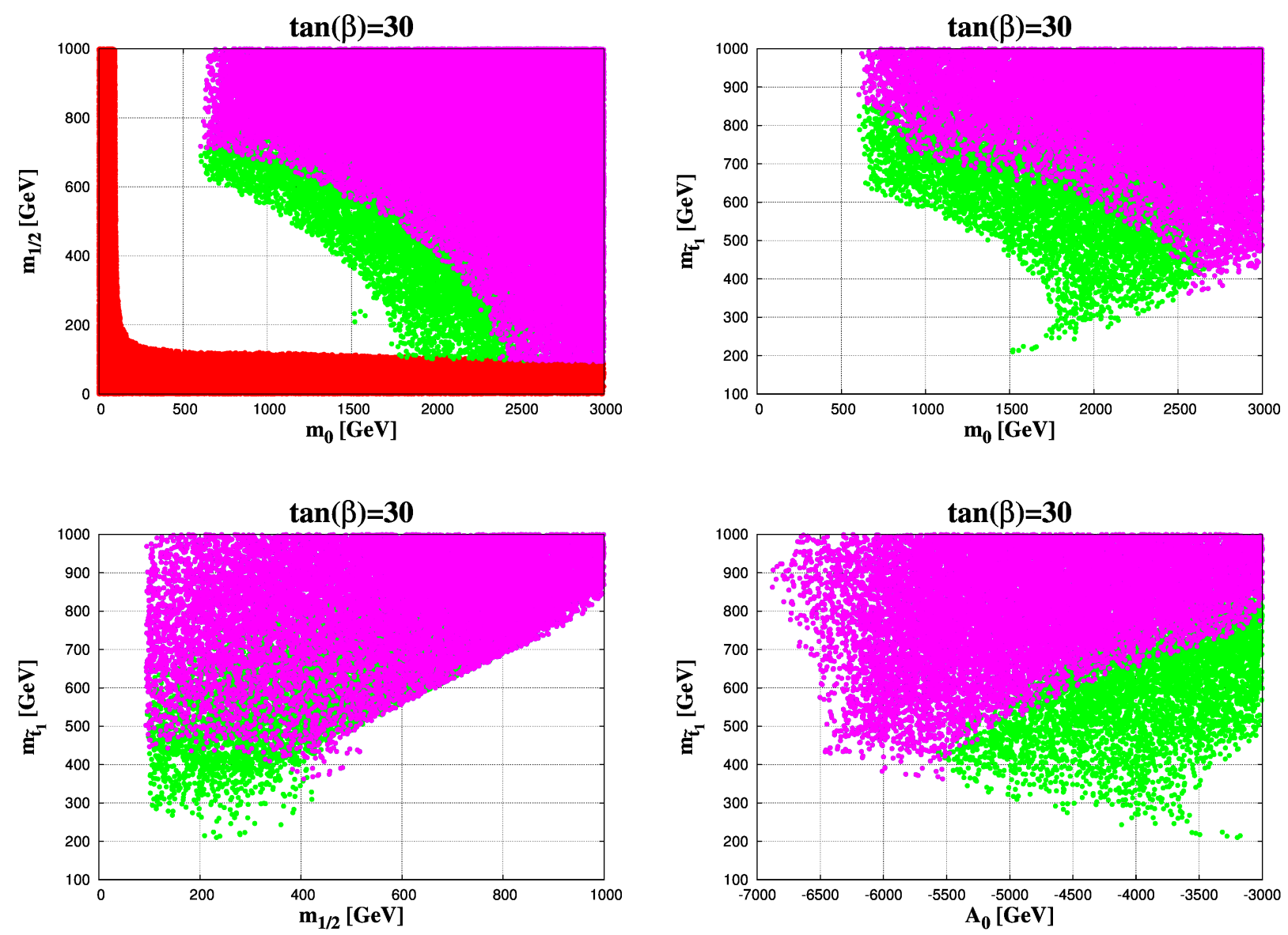

Figure 2: The allowed parameter points in the $m_{0}-m_{1 / 2}$ (top left panel), $m_{0}-m_{\tilde{t}_{1}}$ (top right panel), $m_{1 / 2}-m_{\tilde{t}_{1}}$ (middle left panel), $A_{0}-m_{\tilde{t}_{1}}$ (middle right panel), in the CMSSM scenario for $\tan \beta=30$. The colour code is the same as in Fig. 1.

Corresponding to the signal the principal SM backgrounds that can mimic the signal process are:

- QCD : The final state is swamped by the QCD dijet events, since the QCD cross section at hadron colliders is enormous. The $p_{T}{ }_{T}$ source in this case comes from semileptonic B-decays. There are non physics sources due to mismeasurement of jets, detector noise which are out of the scope of this study.

- $\mathbf{Z}(\rightarrow v \bar{v})+$ jets : This makes up the irreducible part of our background that looks exactly like the signal. Although the principal part of this background is $Z+2$ jets, contribution from higher jet multiplicities are not negligible if some of the jets are not identified.

- $\mathbf{W}(\rightarrow 1 \bar{v})+$ jets : This process contributes dominantly to the background when the lepton is not identified. Since the cross section for $\mathrm{W}+$ jets is rather large this also contributes significantly to the background.

- $\overline{\mathrm{t}}$ : This is primarily dominant when either the leptons from the $\mathrm{W}$ decay and/or some of the final state jets are not identified leading to the same configuration as the signal. 


\begin{tabular}{|c|c|c|c|c|c|c|c|c|c|c|c|c|c|}
\hline & $A_{t}$ & $\tan \beta$ & $\mu$ & $M_{1}$ & $m_{H}$ & $m_{\widetilde{Q}_{3}}$ & $m_{\tilde{t}_{R}}$ & $m_{\tilde{b}_{R}}$ & $m_{\tilde{t}_{1}}$ & $m_{\chi_{1}^{0}}$ & $\Delta m$ & $\begin{array}{c}\mathrm{BR}\left(\widetilde{t}_{1} \rightarrow c \chi_{1}^{0}\right) \\
\%\end{array}$ & $\begin{array}{c}\mathrm{BR}\left(\tilde{t}_{1} \rightarrow b f f^{\prime} \chi_{1}^{0}\right) \\
\%\end{array}$ \\
\hline P3 & -1900 & 10 & 800 & 280 & 123.0 & 380 & 1500 & 2000 & 355 & 285 & 70 & 98 & 2 \\
\hline P4 & -2800 & 10 & 800 & 425 & 124.6 & 450 & 1800 & 1800 & 458 & 432 & 26 & 96 & 3 \\
\hline P5 & -2800 & 10 & 800 & 510 & 126.6 & 530 & 1800 & 1800 & 548 & 517 & 31 & 95 & 4 \\
\hline P6 & -2800 & 30 & 800 & 425 & 128 & 500 & 1800 & 1800 & 520 & 432 & 88 & 98 & 2 \\
\hline
\end{tabular}

Table 2: Masses of some of the sparticles for the benchmark points in the pMSSM scenario. In all cases , the remaining parameters are as described in the text. All energy units are in $\mathrm{GeV}$.

- WW : This process contributes to the background when one W decays hadronically while the other leptonically.

- WZ : This again contributes substantially to the background when $\mathrm{W}$ decays leptonically and $\mathrm{Z}$ decays hadronically with the lepton not being identified, or when $\mathrm{Z}$ decays to $v \bar{v}$ and W hadronically.

- $\mathbf{Z Z}$ : This irreducible background mimics the signal in the situation $\mathrm{Z}(\rightarrow v \bar{v}) \mathrm{Z}(\rightarrow q \bar{q})$.

We simulate the signal and the background processes $t \bar{t}$, WW,WZ,ZZ using PYTHIA6 [104]. For the background processes W/Z+jets, parton level events are generated using ALPGEN [105] and subsequently passed on to PYTHIA6 for showering and hadronization. Jets are reconstructed using FastJet [106] with anti-k $\mathrm{k}_{\mathrm{T}}$ [107] algorithm setting a size parameter $\mathrm{R}=0.5$. Jets are selected with the following criteria,

$$
\mathrm{p}_{\mathrm{T}}^{\mathrm{j}} \geq 30(60) \mathrm{GeV} \forall 8(13) \mathrm{TeV},|\eta| \leq 3 .
$$

MLM matching [108] is performed while showering parton level events using PYTHIA for $\mathrm{W} / \mathrm{Z}+$ jets with a matching cone of $\Delta \mathrm{R}=0.7$ and a jet $p_{T}$ threshold of $30 \mathrm{GeV}$ within $|\eta| \leq 2.5$. We use CTEQ6L [109] as parton density function(PDF) from the LHAPDF [110] package and set $Q^{2}=\hat{s}$. Leptons are selected with,

$$
p_{T}^{l} \geq 10 \mathrm{GeV},|\eta| \leq 2.5
$$

which are used to veto events.

In order to suppress these backgrounds, in particular the large QCD di-jet background, we use kinematic variable,

$$
\alpha_{\mathrm{T}}=\mathrm{p}_{\mathrm{T}}^{\mathrm{j}_{2}} / \mathrm{m}_{\mathrm{T}}^{\mathrm{jj}}
$$

where $\mathrm{p}_{\mathrm{T}}^{\mathrm{j}_{2}}$ is the transverse momentum of the second hardest jet and $\mathrm{m}_{\mathrm{T}}^{\mathrm{jj}}$ is the transverse mass of the two jet system [111]. It can be observed that for pure dijet events, without any hard $\mathrm{p}_{\mathrm{T}}$ like QCD, the jets are back to back in the transverse plane. Therefore the minimum value of $m_{T}^{j j}$ in the limit when jet masses can be ignored turns out to be $2 p_{T}^{j}$ and thus the distribution of $\alpha_{\mathrm{T}}$ has a sharp end point at 0.5. However for dijet events in association with a significant amount of $p_{T}$, as is the case for the signal in Eq. 4, the two jets are not back to back, leading to large values of $\alpha_{T}$. We present the $\alpha_{T}$ distribution for signal and background in Fig. 3 subject to the jet selection cuts ( Eq. 8 ) along with lepton veto. The signal process is for P2 in Table 1 with $m_{\tilde{t}_{1}}, m_{\chi_{1}^{0}}$ masses of 331 and $306 \mathrm{GeV}$ respectively. The number of events generated to construct these plots correspond to the 
fourth column in Table 3 ( $8 \mathrm{TeV})$. It can be seen in the figure, that as predicted, the QCD process has a sharp fall at $\approx 0.5$ and therefore we impose a selection cut [111],

$$
\alpha_{T}>0.55 \text {. }
$$

In addition we also use the kinematic variable $M_{T 2}[112,113]$ defined as,

$$
\mathrm{M}_{\mathrm{T} 2}\left(\mathrm{j}_{1}, \mathrm{j}_{2}, \mathrm{p}_{\mathrm{T}}\right)=\min \left[\max \left\{\mathrm{M}_{\mathrm{T}}\left(\mathrm{j}_{1}, \chi\right), \mathrm{M}_{\mathrm{T}}\left(\mathrm{j}_{2}, \chi\right)\right\}\right],
$$

the minimization being performed over $p_{T}^{1}+p_{T}^{2}=p_{T}^{\prime}$ where $p_{T}^{1}, p_{T}^{2}$ are all possible partitions of invisible transverse momentum $\left(\not{ }_{T}\right)$, which is due to the presence of LSP for signal and neutrinos for SM backgrounds. Here $\chi$ is the invisible particle whose mass $\left(M_{\chi}\right)$ is an unknown parameter. The kinematic variable transverse $\operatorname{mass}\left(M_{T}\right)$ between the jet and the accompanying missing particle is,

$$
M_{T}^{2}=M_{j}^{2}+M_{\chi}^{2}+2\left(E_{T}^{j} E_{T}^{\chi}-\vec{p}_{T}^{j} \cdot \vec{p}_{T}^{\chi}\right),
$$

where $\vec{p}_{T}^{j}$ is the transverse momentum vector of the jet and $E_{T}^{j}$ the corresponding energy, while $\vec{p}_{T}^{\chi}$ is the missing transverse momentum $\left(p_{T}{ }_{T}\right)$ vector.

Since the maximum value of $M_{T}$ is restricted to the mass of the parent particle, $M_{T 2}$ is also expected to be bounded by the respective parent particle mass. Here $M_{T 2}$ is calculated by setting $M_{\chi}=0$ without any loss of generality [114]. This assumption is clearly valid for SM processes where the missing momentum is mainly due to neutrinos. Furthermore, we found no significant difference to the population of events near the end points for the signal with massive $\chi$ when we make this assumption. However, there may be a difference in acceptance for the two cases which we will consider as a systematic uncertainty in the acceptance efficiency. In the SUSY processes where the parent particle $\left(\widetilde{t}_{1}\right)$ is heavier than SM particles, the tail in the $M_{T 2}$ distribution is expected to extend up to a larger value. This variable thus provides an excellent handle to suppress the remainder of the background rates.

In Figure 4, the signal and background distributions for $\mathrm{M}_{\mathrm{T} 2}$ are displayed for 8 (left) and 13(right) TeV LHC energies. The distribution is subject to a di-jet criteria along with a lepton veto with jet and lepton selection criteria(Eq.8 and 9). The signal distribution displayed in the figure corresponds to the benchmark P2 in Table 1. The number of events generated to construct these plots correspond to the fourth column in Tables $3(8 \mathrm{TeV})$ and $4(13 \mathrm{TeV})$.

We observe that for the signal process at $8 \mathrm{TeV}$ the tail extends beyond the background processes and further reaches beyond the stop mass while for the background the end point of the distributions correspond to lower values of $\mathrm{M}_{\mathrm{T} 2}$. As the missing energy in $\mathrm{Z}+\mathrm{jets}$ comes from $\mathrm{Z}$ decaying to a pair of neutrinos from the same side of the configuration, the $\mathrm{M}_{\mathrm{T} 2}$ distribution is not expected to have an end point at the $\mathrm{Z}$ mass which is reflected in this plot [114]. In case of $t \bar{t}$ background and the signal also, the distribution extends beyond the expected end point at the parent particle mass. The contribution of events in this region is dominantly due to the jets from hard radiations, in particular from final state radiations [114]. This phenomena is more evident at $13 \mathrm{TeV}$ energy where objects are kinematically heavily boosted. By observing the distributions, we apply a cut at,

$$
M_{T 2}>130 \mathrm{GeV}(250 \mathrm{GeV}) \text { for } 8(13) \mathrm{TeV} \text {. }
$$

This fact can be exploited to suppress backgrounds when the signal is in unfavourable condition kinematically (eg. for small $\Delta m$ ). For example, for very small $\Delta m, \tilde{t}_{1}$ mostly decays invisibly, and hence $M_{T 2}$ constructed out of this hard $p_{T}$ and jets originating from initial and final state radiation(ISR/FSR) which are un-correlated with the $\not_{T}^{\prime}$ has a much larger tail. For larger values 


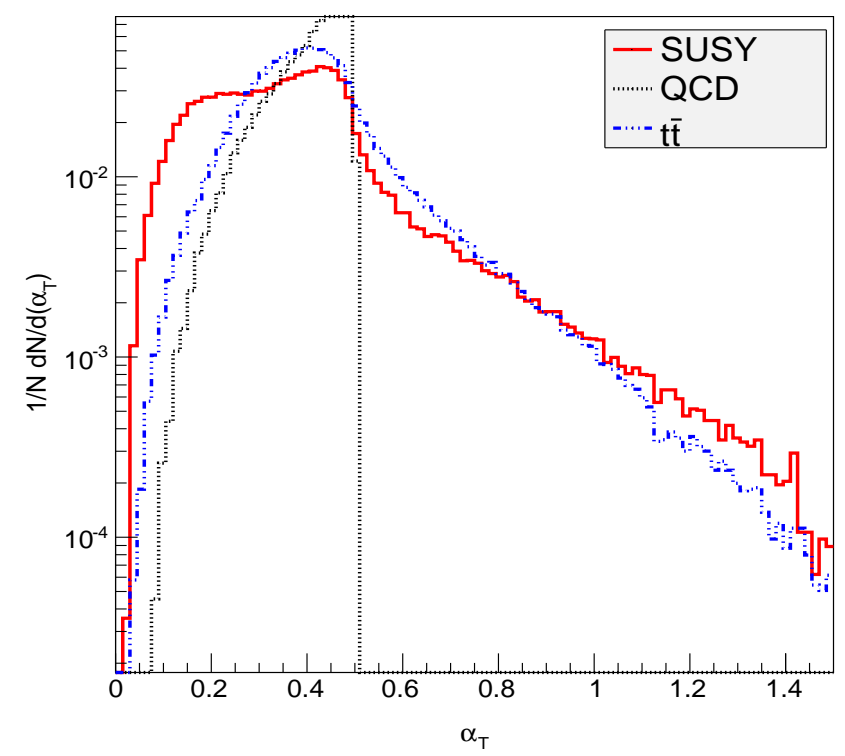

Figure 3: $\alpha_{T}$ distribution for signal and background at $8 \mathrm{TeV}$ LHC energy. The signal corresponds to P2 from Table 1. The number of events generated correspond to the fourth column in Table 3.

of $\Delta m$, the longer tail is not observed due to the fact that $\tilde{t}_{1}$ will have both visible and the invisible (LSPs) decay products for which the which momenta are correlated. In Fig.5 we present the distribution for these two cases, with $\Delta m=10$ and $140 \mathrm{GeV}$. Clearly $M_{T 2}$ gives some handle to recover sensitivity for the low $\Delta m$ scenario. It is to be noted here also that for the case $\Delta m=140$ $\mathrm{GeV}$, the decay $\tilde{t}_{1} \rightarrow b W \chi_{1}^{0}$ may open up, competing with the flavor violating decay mode. Clearly the flavour violating decay mode with very low $\Delta m$ have some benefits due to ISR/FSR effects. However, it is clear that these effects have a dependence on the models employed in the event generators. Therefore, in order to understand its effect in our signal sensitivity in a more precise manner one needs to do more detailed investigation which is postponed to a future work.

Finally, considering signal and background characteristics, we use the following cuts to suppress the backgrounds.

- Lepton Veto (LV) : In the signal process leptonic activity is absent. However background processes like $t \bar{t}, \mathrm{~W}+\mathrm{jets}, \mathrm{WW}, \mathrm{WZ}$ contain a significant fraction of leptons from $\mathrm{W} / \mathrm{Z}$ decays accompanied by $p_{T}^{\prime}$. The use of lepton veto thus helps to suppress the backgrounds efficiently. Leptons are selected using cuts described in Eq. 8.

- 2 jets (2J) : We select exclusively dijets with the jet $\mathrm{p}_{\mathrm{T}}$ thresholds as described in Eq. 9. Note that after the lepton veto, the $t \bar{t}$ background contribution is expected to be rich in hadronic activity and have more than 2 jets in the event. Hence a strict imposition of the dijet criteria is expected to reduce the background coming from the $t \bar{t}, \mathrm{~W} / \mathrm{Z}+$ jet processes.

- b-jet veto (bJV) : This veto is extremely efficient in suppressing the top background. The bjet identification is implemented by performing a matching of the jets with $b$ quarks assuming a matching cone $\Delta R(b, j)=0.2$.

- $\alpha_{\mathrm{T}} \geq 0.55$ as discussed in Eq. 11 . 

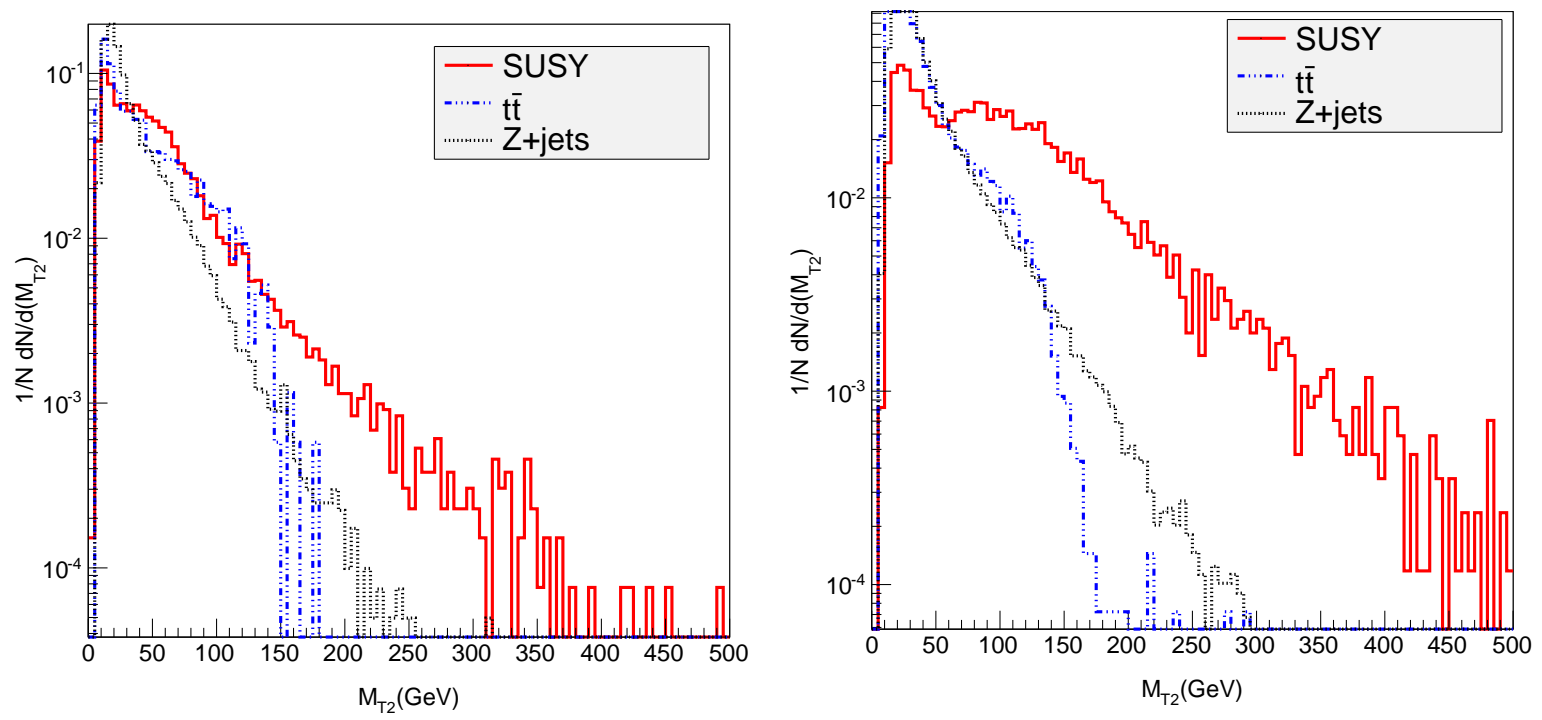

Figure 4: $M_{T_{2}}$ distribution for signal and background for $8 \mathrm{TeV}$ (left) and $13 \mathrm{TeV}$ (right). The signal corresponds to P2 in Table 1. The number of events generated correspond to the fourth column in Tables $3(8 \mathrm{TeV})$ and 4 (13 TeV).

- $\mathrm{M}_{\mathrm{T} 2} \geq 130 \mathrm{GeV}(250 \mathrm{GeV}$ for $13 \mathrm{TeV})$ as given by Eq. 14 .

- $\mathrm{p}_{\mathrm{T}} / \mathrm{H}_{\mathrm{T}} \leq 0.9$ : For signal processes we expect this ratio to be less than 1, while in background processes this is expected to be close to 1 . The difference in azimuthal angle between the two jet system and missing energy in signal and background being primarily responsible for this behaviour. We find that this selection is extremely effective in suppressing the $t \bar{t}$ background.

At the end, we explore the possibility of an improvement by somehow tagging charm jets which are a part of the signal. The identification of charm jet is quite challenging. Recently however, attempts have been made to measure $\mathrm{W}+\mathrm{c}$ jets cross-section where $\mathrm{c}$-jets are identified [115]. Note that the channel $\tilde{t}_{1} \rightarrow c \chi_{1}^{0}$, has been recently searched by ATLAS by trying to identify charm jets in the final state [116]. Although the method we employ is rather simplified, it still points out that charm like jet identification can prove to be extremely effective in this case. To identify charm jets we match jets with charm quarks, using a matching cone of $R=0.2$. To find the presence of charm jets one can further check the presence of D-meson among the jet constituents, which we postpone to a future work.

\section{Results}

Simulating the signal and background processes using the selection cuts as described above, we present results for 8 and $13 \mathrm{TeV}$ LHC energies in Tables 3 and 4 respectively. The first four columns present the processes(Proc), the masses of the lightest $\operatorname{stop}\left(m_{\tilde{t}_{1}}\right)$ and $\operatorname{LSP}\left(m_{\chi_{1}^{0}}\right)$, the cross section(C.S), and the number of events generated $(\mathrm{N})$ for each process respectively. We compute the next to leading order signal cross section using PROSPINO [117]. The subsequent columns display the cumulative effects of cuts, as normalized cross sections (production cross section multiplied by cut efficiency) . In the penultimate column the cross sections after all cuts are presented. 


\begin{tabular}{|c|c|c|c|c|c|c|c|c|}
\hline Proc & $\begin{array}{c}m_{\tilde{t}_{1}}, m_{\chi_{1}^{0}} \\
\mathrm{GeV}\end{array}$ & C.S & $\mathrm{N}$ & $\mathrm{LJV}+2 \mathrm{~J}+\mathrm{bJV}$ & $\begin{array}{c}\alpha_{T} \\
\geq 0.55\end{array}$ & $\begin{array}{c}M_{T 2} \geq \\
130\end{array}$ & $\begin{aligned} & \frac{\eta_{T}}{H_{T}} \\
& \leq 0.9\end{aligned}$ & $\begin{array}{c}\text { C.S } \\
\text { (c-like) }\end{array}$ \\
\hline P1 & 241,198 & 6330 & $0.5 \mathrm{M}$ & 1996.2 & 262.3 & 4.7 & 2.1 & 0.45 \\
\hline P2 & 331,306 & 1060 & $0.5 \mathrm{M}$ & 227.4 & 28.1 & 2.3 & 0.9 & 0.1 \\
\hline P3 & 355,285 & 700 & $0.5 \mathrm{M}$ & 262 & 46 & 0.6 & 0.25 & 0.12 \\
\hline P4 & 458,432 & 150 & $0.5 \mathrm{M}$ & 33.1 & 4.2 & 0.3 & 0.14 & 0.01 \\
\hline P5 & 548,517 & 45 & $0.5 \mathrm{M}$ & 11.5 & 1.42 & 0.1 & 0.04 & 0.007 \\
\hline P6 & 520,432 & 63 & $0.5 \mathrm{M}$ & 23.5 & 4.1 & 0.085 & 0.04 & 0.02 \\
\hline $\mathrm{t} \overline{\mathrm{t}}-5-200$ & & 85000 & $2 \mathrm{M}$ & 6063.4 & 80.24 & 8.9 & 0.46 & 0.17 \\
\hline $\mathrm{t} \overline{\mathrm{t}}-200-500$ & & 9500 & $0.5 \mathrm{M}$ & 13.9 & 3.9 & 1.5 & 0.38 & 1 \\
\hline$\overline{\mathrm{t}} \mathrm{t}-500-\infty$ & & 130 & $0.5 \mathrm{M}$ & 2.1 & 0.005 & 0.003 & 0.003 & $<1$ \\
\hline qcd-300-500 & & $1.3 \times 10^{6}$ & $3 \mathrm{M}$ & 37512.7 & $<1$ & $<1$ & $<1$ & $<1$ \\
\hline WW & & 35000 & $0.5 \mathrm{M}$ & 7462.2 & 380.2 & 2.94 & 0.84 & 0.14 \\
\hline WZ & & 13000 & $0.5 \mathrm{M}$ & 2547.8 & 189.3 & 3.1 & 0.76 & 0.21 \\
\hline $\mathrm{ZZ}$ & & 5400 & $0.5 \mathrm{M}$ & 1050.1 & 78.12 & 1.62 & 0.3 & 0.02 \\
\hline $\mathrm{Z}(\rightarrow v \bar{v})+2 \mathrm{jet}$ & & $10^{5}$ & 320910 & 71215.2 & 6877.4 & 67.07 & 29.4 & $<1$ \\
\hline $\mathrm{Z}(\rightarrow v \bar{v})+3$ jet & & 16500 & 241202 & 5349.6 & 637.0 & 9.9 & 4.5 & 0.13 \\
\hline $\mathrm{Z}(\rightarrow v \bar{v})+4$ jet & & 4240 & 39203 & 361.4 & 41.3 & 0.75 & 0.6 & $<1$ \\
\hline $\mathrm{W}(\rightarrow l v)+2 \mathrm{jet}$ & & $5.8 \times 10^{5}$ & 565087 & 117335.6 & 10752.1 & 63.4 & 35.3 & 2.1 \\
\hline $\mathrm{W}(\rightarrow l v)+3 \mathrm{jet}$ & & $10^{5}$ & 364287 & 14100.0 & 1240.8 & 9.1 & 6.1 & $<1$ \\
\hline $\mathrm{W}(\rightarrow l v)+4 \mathrm{jet}$ & & 16300 & 44238 & 785.6 & 68.9 & 1.5 & 1.1 & $<1$ \\
\hline
\end{tabular}

Table 3: The cross sections(fb) for signal and backgrounds after each cuts for $8 \mathrm{TeV}$ LHC energy. The last two columns present normalized cross section(fb) after all cuts without and with identification of c-like jets respectively. All energy units are in $\mathrm{GeV}$.

The top and the QCD backgrounds are simulated by slicing the entire phase space into $\hat{\mathrm{p}}_{\mathrm{T}}$ bins, where $\hat{p_{T}}$ is the transverse momenta of the produced partons in the partonic frame. In both Tables we notice that the combined effects of lepton veto, $b$-jet veto and the dijet criteria $(L V+2 J+b J V)$ reduces the top background by an enormous amount $(\sim 95 \%)$ while reducing the signal process by about a third. As pointed out earlier, the $\alpha_{\mathrm{T}}$ cut successfully isolates the entire QCD background as expected. For the sake of simplicity we have quoted numbers corresponding to only one $\hat{\mathrm{p}}_{\mathrm{T}}$ bin for QCD. The rest of the backgrounds, particularly from top and the WW/WZ/ZZ are also suppressed by a significant amount, costing a significant fraction of signal cross section as well. The $\mathrm{M}_{\mathrm{T} 2}$ cut, as pointed out, removes a substantial fraction of $t \bar{t}, \mathrm{WW} / \mathrm{WZ} / \mathrm{ZZ}$ as well as $\mathrm{W} / \mathrm{Z}+$ jets processes. Clearly the $M_{T 2}$ cut plays an important role in isolating backgrounds efficiently. Finally the cut $p_{T} / H_{T}$ suppresses the WW/WZ/ZZ backgrounds and brings it down to a negligible level. Even after a huge suppression of the irreducible backgrounds $\mathrm{W} / \mathrm{Z}+2,3$ jets, the remaining fraction is non negligible because of the large production cross section. Note that even after suppressing the SM backgrounds substantially, since signal cross section is miniscule, the prospects of discovering a signal at $8 \mathrm{TeV}$ LHC is very limited. In the last column cross sections are presented requiring that out of the two jets in the final state one is a c-like jet. As mentioned earlier, c-like jets are identified by naively matching partonic c-quarks and reconstructed jets. Clearly, it shows that identification of c-like jets does help in reducing the background to a large extent. This happens as the signal is likely to have a larger fraction of identified charm jets than the background.

At $13 \mathrm{TeV}$ the results improve significantly as can be seen from Table 4. The larger stop pair production cross section significantly helps in enhancing the event rates. On the other hand an increased boost in the system helps to effectively use the $M_{T 2}$ variable by applying a much larger cut value of $250 \mathrm{GeV}$ to isolate the backgrounds. As can be observed from the right panel of Fig 


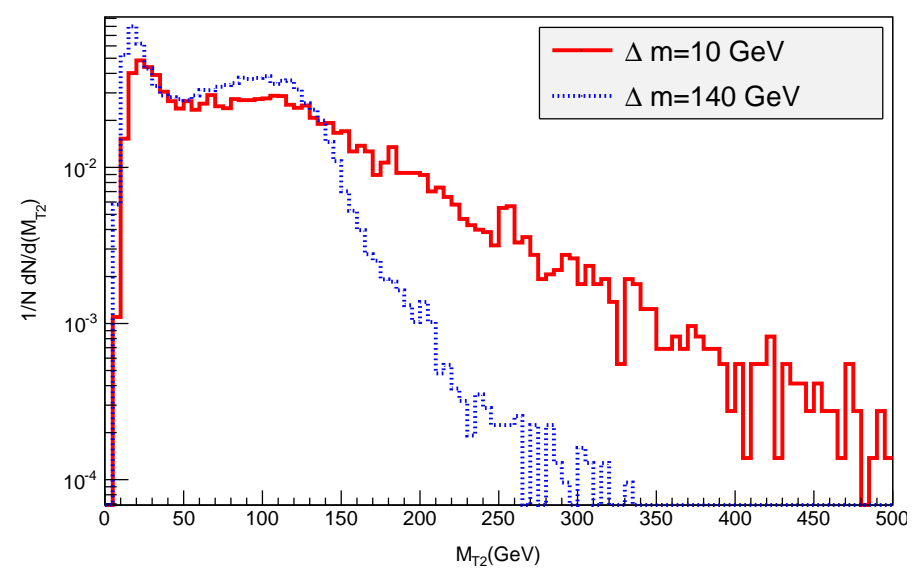

Figure 5: $M_{T 2}$ distribution for two values of $\Delta m$ of $10 \mathrm{GeV}$ for solid(red) and $140 \mathrm{GeV}$ for broken(blue) for a $m_{\tilde{t}_{1}}$ of $240 \mathrm{GeV}$ at $13 \mathrm{TeV}$ LHC energy. $0.5 \mathrm{M}$ events were generated to construct these plots.

4, a cut of $250 \mathrm{GeV}$ is extremely effective in suppressing the irreducible Z+jets background. We observe from the last column of Table 4 that at $13 \mathrm{TeV}$ energy, the signal and background cross sections are comparable as compared to $8 \mathrm{TeV}$ where the background cross sections are dominant.

Table 5 summarizes cross sections of the signal and background after all cuts for 8 and $13 \mathrm{TeV}$ LHC energy. The $t \bar{t}$ cross section has been multiplied in the table by a $\mathrm{k}$ factor of 2 to take into account NLO effects [118]. Note that the k-factors for $\mathrm{W} / \mathrm{Z}+$ jets processes are very close to $1[119,120]$, and hence do not change our results significantly. From Table 5 we find that at $8 \mathrm{TeV}$ the total background is $70.6 \mathrm{fb}$ in which the dominant contribution comes from $\mathrm{W} / \mathrm{Z}+$ jets, while the signal cross section varies from $2.1 \mathrm{fb}$ for P1 to $0.04 \mathrm{fb}$ in P6. Therefore for P1 with $20 \mathrm{fb}^{-1}$ luminosity we obtain $S / \sqrt{B}=1.1$, while the significance drops substantially with the increase of $m_{\tilde{t}_{1}}$. At $13 \mathrm{TeV}$ the total background cross section turns out to be $2.6 \mathrm{fb}$ while the signal cross section varies from $4.4 \mathrm{fb}$ in $\mathrm{P} 1$ to $0.1 \mathrm{fb}$ in P6. Thus for the points $\mathrm{P} 1$ and $\mathrm{P} 2$ we obtain $S / \sqrt{B}=6.1$ and 4.4 respectively for $5 \mathrm{fb}^{-1}$ which implies that $\mathrm{P} 1$ is discoverable while an evidence of a signal can be obtained even with low luminosity options for P2. With $100 \mathrm{fb}^{-1}$ luminosity we find that the benchmarks $\mathrm{P} 3$ and $\mathrm{P} 4$ have significance values of 4.7 and 4.3 respectively.

With this strategy we attempt to explore the sensitivity in $\Delta m$ for various values of $m_{\tilde{t}_{1}}$ at a given luminosity. We present our findings in Fig. 6 where the accessible region below the curves are shown in the $m_{\tilde{t}_{1}}-\Delta m$ plane for two luminosity options $5 \mathrm{fb}^{-1}$ and $100 \mathrm{fb}^{-1}$ with $S \sqrt{B} \geq 5$. This plot is presented with the assumption of $B R\left(\tilde{t}_{1} \rightarrow c \chi_{1}^{0}\right)=100 \%$, holding the rest of the parameters fixed as described in section 2. The plot is obtained by a parameter space scan in the $m_{\tilde{t}_{1}}-\Delta m$ plane with a grid spacing of $20 \mathrm{GeV}$ on the $m_{\tilde{t}_{1}}(\mathrm{X})$-axis and $5 \mathrm{GeV}$ on the $\Delta m(\mathrm{Y})$-axis. We find that even for low luminosity a light stop up to a mass of $\sim 350 \mathrm{GeV}$ could be explored for $\Delta m=20 \mathrm{GeV}$. As mentioned earlier this search strategy is very sensitive to lower values of $\Delta m$ and it is reflected in the figure. For a luminosity of $100 \mathrm{fb}^{-1}$ we find that a light stop up to a mass of $450 \mathrm{GeV}$ can be probed for $\Delta m$ as low as $35 \mathrm{GeV}$. The solid horizontal line demarcates the kinematic region $m_{\tilde{t}_{1}}<m_{t}+m_{\chi_{1}^{0}}$, over which the the decay $\tilde{t}_{1} \rightarrow t \chi_{1}^{0}$ opens up and dominates. 


\begin{tabular}{|c|c|c|c|c|c|c|c|c|}
\hline Proc & $\begin{array}{c}m_{\tilde{t}_{1}}, m_{\chi_{1}^{0}} \\
\mathrm{GeV}\end{array}$ & $\mathrm{C} . \mathrm{S}$ & $\mathrm{N}$ & $\mathrm{LJV}+2 \mathrm{~J}+\mathrm{bJV}$ & $\begin{array}{c}\alpha_{T} \\
\geq 0.55\end{array}$ & $\begin{array}{c}M_{T 2} \geq \\
250\end{array}$ & $\begin{array}{c}p_{T} \bar{H}_{T} \\
\leq 0.9\end{array}$ & $\begin{array}{c}\mathrm{C} . \mathrm{S} \\
(\mathrm{c}-\mathrm{like})\end{array}$ \\
\hline $\mathrm{P} 1$ & 241,198 & 24100 & $0.5 \mathrm{M}$ & 3113.2 & 311.6 & 8.1 & 4.4 & 0.45 \\
$\mathrm{P} 2$ & 331,306 & 4800 & $0.5 \mathrm{M}$ & 440.2 & 82.0 & 5.8 & 3.2 & 0.18 \\
$\mathrm{P} 3$ & 355,285 & 3280 & $0.5 \mathrm{M}$ & 725.5 & 83.27 & 1.95 & 0.7 & 0.14 \\
$\mathrm{P} 4$ & 458,432 & 820 & $0.5 \mathrm{M}$ & 84.7 & 16.8 & 1.63 & 0.75 & 0.03 \\
$\mathrm{P} 5$ & 548,517 & 290 & $0.5 \mathrm{M}$ & 34.2 & 6.2 & 0.5 & 0.29 & 0.023 \\
$\mathrm{P} 6$ & 520,432 & 400 & $0.5 \mathrm{M}$ & 121 & 17.8 & 0.29 & 0.1 & 0.04 \\
\hline $\mathrm{t} \overline{\mathrm{t}}-5-200$ & & 291,000 & $4 \mathrm{M}$ & 18690.2 & 2011.1 & 0.15 & 0.15 & $<1$ \\
$\mathrm{tt}-200-500$ & & 39800 & $0.5 \mathrm{M}$ & 492.2 & 85.2 & $<1$ & $<1$ & $<1$ \\
$\mathrm{t} \overline{\mathrm{t}}-500-\infty$ & 900 & $0.5 \mathrm{M}$ & 12.4 & 0.13 & $<1$ & $<1$ & $<1$ \\
$\mathrm{WW}$ & & 69800 & $1 \mathrm{M}$ & 11858.3 & 376.2 & 0.14 & $<1$ & $<1$ \\
$\mathrm{WZ}$ & & 26300 & $1 \mathrm{M}$ & 4144.9 & 231.5 & 0.5 & 0.15 & $<1$ \\
$\mathrm{ZZ}$ & & 10900 & $1 \mathrm{M}$ & 1812.6 & 128.9 & 0.46 & 0.06 & $<1$ \\
\hline $\mathrm{Z}(\rightarrow v \bar{v})+2 \mathrm{jet}$ & & 241,800 & $2,605,885$ & 34572.5 & 2643.4 & 12.4 & 1.3 & $<1$ \\
$\mathrm{Z}(\rightarrow v \bar{v})+3$ jet & & 48000 & 418,467 & 14282.9 & 115.9 & 3.15 & 0.45 & $<1$ \\
$\mathrm{Z}(\rightarrow v \bar{v})+4$ jet & & 11200 & 48473 & 3477.7 & 294.1 & 3.1 & 0.35 & $<1$ \\
$\mathrm{~W}(\rightarrow l v)+2$ jet & & $1,185,000$ & $1,763,283$ & 76017.1 & 3529.8 & 8.4 & $<1$ & $<1$ \\
$\mathrm{~W}(\rightarrow l v)+3$ jet & & 229,000 & 476,382 & 27524.6 & 1490.2 & 3.0 & $<1$ & $<1$ \\
$\mathrm{~W}(\rightarrow l v)+4$ jet & & 47200 & 103,178 & 6278.9 & 400.1 & 1.2 & $<1$ & $<1$ \\
\hline
\end{tabular}

Table 4: Same as Table 3 but for 13 TeV LHC.

\begin{tabular}{|c|c|c|c|c|c|c|c|}
\hline & P1 & P2 & P3 & P4 & P5 & P6 & Total Bg \\
\hline$m_{\tilde{t}_{1}}, m_{\tilde{\chi}_{1}^{0}}(\mathrm{GeV})$ & 241,198 & 331,306 & 355,285 & 458,432 & 548,517 & 520,432 & \\
\hline $8 \mathrm{TeV}$ & 2.1 & 0.9 & 0.25 & 0.14 & 0.04 & 0.04 & 70.6 \\
$S / \sqrt{B}\left(20 \mathrm{fb}^{-1}\right)$ & 1.1 & 0.5 & 0.13 & 0.07 & 0.02 & 0.02 & \\
\hline $13 \mathrm{TeV}$ & 4.4 & 3.2 & 0.7 & 0.75 & 0.29 & 0.1 & 2.6 \\
$S / \sqrt{B}\left(30 \mathrm{fb}^{-1}\right)$ & 15 & 11 & 2.4 & 2.5 & 1 & 0.3 & \\
\hline
\end{tabular}

Table 5: The signal and backgrounds cross sections(cs) for benchmark points. The signal significances $(S / \sqrt{B})$ for different energies and luminosities are also shown.

\subsection{Implications for dark matter}

In scenarios with a small stop-neutralino mass splitting and a bino $(\tilde{B})$ LSP, stop-coannihilation can play an important role in determining the relic dark matter abundance. This is the case especially for small $\Delta m$, i.e, where the decay $\tilde{t}_{1} \rightarrow c \tilde{\chi}_{1}^{0}$ is dominant. Hence it is important to investigate the implications of our studies for probing the stop coannihilation scenario at the LHC.

The relic density crucially depends on the stop-neutralino mass difference as well as on other parameters that will be discussed in the next paragraph. For the benchmarks considered, the value of the relic density as shown in Table 6 is either above $(\mathrm{P} 1, \mathrm{P} 3, \mathrm{P} 6)$ or below $(\mathrm{P} 2, \mathrm{P} 4, \mathrm{P} 5)$ the central value for PLANCK, $\Omega_{D M} h^{2}=0.1199$ [121]. However it is well known that the value of the relic density in coannihilation scenarios depends critically on the NLSP-LSP mass difference, hence a small decrease (increase) in the stop-neutralino mass difference will lead to a large decrease (increase) in the value of the relic density. We have therefore searched for modified benchmarks for which the relic density was in agreement with the central value of PLANCK. For this, we vary only the mass of the lightest neutralino by changing $M_{1}$, while keeping all other parameters of each benchmark P1-P6 to their value at the EWSB scale. The modified benchmarks, P1'-P6', with 


\begin{tabular}{|c|c|c|c|c|c|c|}
\hline & $\mathrm{P} 1$ & $\mathrm{P} 2$ & $\mathrm{P} 3$ & $\mathrm{P} 4$ & $\mathrm{P} 5$ & $\mathrm{P} 6$ \\
\hline$m_{\tilde{t}_{1}}, m_{\tilde{\chi}_{1}^{0}}(\mathrm{GeV})$ & 241,198 & 331,306 & 355,285 & 458,432 & 548,517 & 520,432 \\
$\Omega h^{2}$ & 0.17 & 0.04 & 1.9 & 0.04 & 0.06 & 0.59 \\
\hline \hline & $\mathrm{P} 1$ & $\mathrm{P} 2$ & $\mathrm{P} 3$ & $\mathrm{P} 4$ & $\mathrm{P} 5$ & $\mathrm{P} 6$ \\
\hline$m_{\tilde{t}_{1}}, m_{\tilde{\chi}_{1}^{0}}(\mathrm{GeV})$ & 241,205 & 331,294 & 355,315 & 458,420 & 548,508 & 520,479 \\
$\Omega h^{2}$ & 0.119 & 0.119 & 0.119 & 0.119 & 0.119 & 0.119 \\
$\Delta m(\mathrm{GeV})$ & 36 & 37 & 40 & 38 & 40 & 41 \\
\hline
\end{tabular}

Table 6: Relic density for the benchmarks P1-P6 and the modified benchmarks P1'-P6'

the corresponding stop and neutralino masses are listed in Table 6. The relic density is calculated using micrOMEGAs3 [122]. Furthermore, their position in the $\Delta m-m_{\tilde{t}_{1}}$ plane is displayed in Figure 6. We find that for the benchmark points that satisfy the PLANCK constraint our search strategy works very well indeed. We achieve reasonable sensitivity for stop masses below $400 \mathrm{GeV}$ with an integrated luminosity $\mathscr{L}=100 \mathrm{fb}^{-1}$ at $13 \mathrm{TeV}$ LHC as can be seen in Fig. 6. In fact the $5 \sigma$ significance contours for $\mathscr{L}=5 \mathrm{fb}^{-1}$ even covers the relevant $\Delta m$ for stop masses below 280 $\mathrm{GeV}$.

A few comments are in order to ascertain the generality of this result since the relic density depends not only on the stop-neutralino mass difference but also on the nature of the neutralino LSP, the nature of $\tilde{t}_{1}$ (whether it is dominantly LH or RH), and on the value of $M_{A}$. First note that the mass splittings associated with the modified benchmarks of Table 6 are typical of scenarios where the LSP is a bino, and these are precisely the ones where stop coannihilation plays an important role in obtaining a low enough relic density. Second, the mass splitting required to satisfy the PLANCK constraint - for a given stop mass - should depend on whether the stop is LH (P3'-P6') or RH (P1'-P2'). The reason is the following: co-annihilation processes such $\tilde{\chi}_{1} \tilde{t}_{1} \rightarrow t g$, th have a larger cross-section for a RH stop than for a LH stop of the same mass since the coupling to the bino LSP is proportional to the top hypercharge (which is larger for the RH top/stop), hence one would expect the required $\Delta m$ to be larger for a RH stop. Furthermore the QCD processes involving pairs of squarks $\tilde{t}_{1} \tilde{t}_{1} \rightarrow t t, \tilde{t}_{1} \tilde{b}_{1} \rightarrow t b \ldots$ which are more important for LH stops involve two Boltzmann suppression factors ${ }^{2}$, therefore the mass splitting required is smaller. However since the Boltzmann factor varies rapidly with $\Delta m$, in the end there is only a few GeV differences between the case of the RH and LH stop. For example for benchmark P2', the required mass splitting would be $\Delta m=29 \mathrm{GeV}$ for a dominantly LH stop instead of $\Delta m=37 \mathrm{GeV}$ for a RH one. Finally, the pseudoscalar mass, $M_{A}$, can also be a relevant parameter. For P1' and P2' it is set by CMSSM boundary conditions and is rather high hence plays no role in neutralino annihilation while for P3'-P6', it is set to $500 \mathrm{GeV}$. A higher value of $M_{A}$ would not affect our collider search strategy and the relevant branching ratios of $\tilde{t}_{1} \rightarrow c \chi_{1}^{0}$. However it would imply smaller mass differences than the ones listed in Table 6, and hence would easily be covered by our search strategy. We can therefore safely conclude that the channel investigated here can probe the stopcoannihilation scenario for stop masses up to at least $400 \mathrm{GeV}$.

\footnotetext{
${ }^{2}$ The Boltzmann factor is $e^{-\Delta m} / T_{f}$ for each coannihilating particle and $T_{f}$ is the freeze-out temperature.
} 


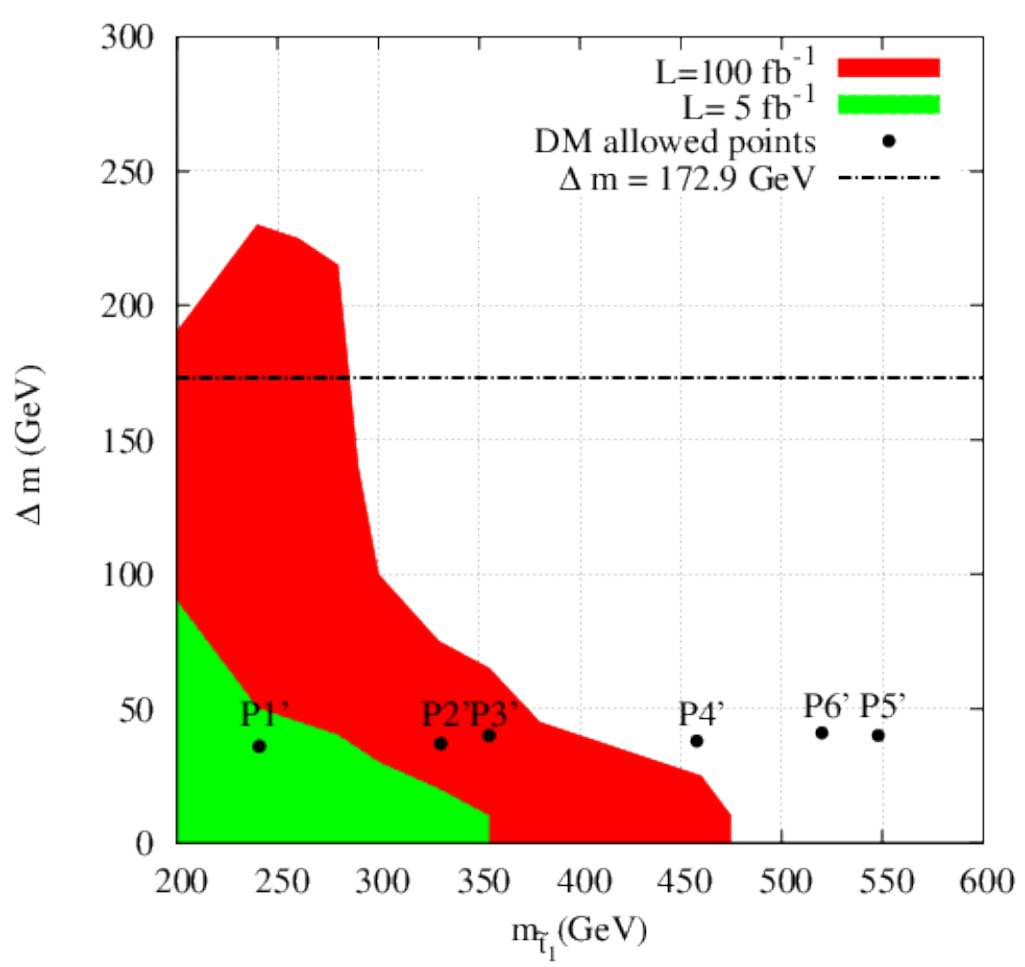

Figure 6: The $5 \sigma$ significance contours for $\mathscr{L}=5 \mathrm{fb}^{-1}$ \{green(light solid) $\}$, and for $\mathscr{L}=100 \mathrm{fb} b^{-1}\{$ red(dark solid) $\}$ luminosity assuming $\tilde{t}_{1} \rightarrow c \chi_{1}^{0}$ to be $100 \%$ for $13 \mathrm{TeV}$ LHC energy. The black (broken) line corresponds to $m_{t}=172.9 \mathrm{GeV}$ and is the kinematic limit for $\tilde{t}_{1} \rightarrow t+\chi_{1}^{0}$. The dark matter allowed points P1'-P6' corresponding to Table 6 are denoted by the black (solid) dots.

\section{Conclusion}

In this study we perform a comprehensive analysis of the collider search prospects of the flavor violating decay of the stop quark, namely $\tilde{t}_{1} \rightarrow c \chi_{1}^{0}$. Such a scenario is well motivated in the context of natural SUSY as well as from the dark matter perspective of stop co-annihilation. It had been earlier observed that this channel is rather difficult to probe due to the low mass difference between the stop quark and the lightest neutralino. The principle background to this channel arises from QCD, $t \bar{t}$ and $\mathrm{Z}(v \bar{v}) / \mathrm{W}(\rightarrow l v)+$ jets final state. We use the kinematic variables $\alpha_{T}$ and $M_{T 2}$ to effectively suppress these at 8 and $13 \mathrm{TeV}$ LHC. At $8 \mathrm{TeV}$, the level of background is still high and we are limited by low stop pair production cross section. We find that our strategy is far more effective at $13 \mathrm{TeV}$ due to the increase in cross section and efficient use of the kinematic variables. We observe that it is possible to discover light stop quarks up to a mass of $\sim 450 \mathrm{GeV}$ with 100 $f b^{-1}$ luminosity at $13 \mathrm{TeV}$ LHC energy for low values of $\Delta \mathrm{m}$ and even for the case when the $\tilde{t}_{1}$ and $\chi_{1}^{0}$ are almost degenerate. We observe that for very low $\Delta m$ case, the loss of acceptance because of soft visible particles in the final states is compensated by ISR/FSR effects through $\mathrm{M}_{\mathrm{T} 2}$ selection. The result improves significantly when one attempts identifying charm jets both at 8 and $13 \mathrm{TeV}$ LHC. We also show the discoverable region in $m_{\tilde{t}_{1}}-\Delta m$ plane assuming the branching ration of $\tilde{t}_{1} \rightarrow c \chi_{1}^{0}$ to be $100 \%$. This is an useful information in the context of DM via stop co-annihilation. Our analysis shows that a good region of the parameter space relevant for the stop - coannihilation scenario can be probed at $13 \mathrm{TeV}$ LHC energy. 


\section{Acknowledgements}

The authors would like to thank the organizers of WHEPP-XII held at Mahabaleswar,India in 2012, and the BSM working group, where this project was initiated. GB thanks the Aspen Center for Physics for hospitality during the final stage of this work. Partial funding by the French ANR DMAstroLHC is gratefully acknowledged. The research by DG leading to these results has received funding from the European Research Council under the European Unions Seventh Framework Programme (FP/2007-2013) / ERC Grant Agreement n.279972. RMG wishes to acknowledge the Department of Science and Technology of India, for financial support under the J.C. Bose Fellowship scheme under grant no. SR/S2/JCB-64/2 007.

\section{References}

[1] CMS Collaboration, S. Chatrchyan et al., "Observation of a new boson at a mass of $125 \mathrm{GeV}$ with the CMS experiment at the LHC," Phys.Lett. B716 (2012) 30-61, arXiv:1207.7235 [hep-ex].

[2] ATLAS Collaboration, G. Aad et al., "Observation of a new particle in the search for the Standard Model Higgs boson with the ATLAS detector at the LHC," Phys.Lett. B716 (2012) 1-29, arXiv:1207.7214 [hep-ex].

[3] H. Baer, V. Barger, and A. Mustafayev, "Implications of a $125 \mathrm{GeV}$ Higgs scalar for LHC SUSY and neutralino dark matter searches," Phys.Rev. D85 (2012) 075010, arXiv:1112.3017 [hep-ph].

[4] S. Akula, B. Altunkaynak, D. Feldman, P. Nath, and G. Peim, "Higgs Boson Mass Predictions in SUGRA Unification, Recent LHC-7 Results, and Dark Matter," Phys.Rev. D85 (2012) 075001, arXiv:1112.3645 [hep-ph].

[5] J. L. Feng, K. T. Matchev, and D. Sanford, "Focus Point Supersymmetry Redux," Phys.Rev. D85 (2012) 075007, arXiv:1112.3021 [hep-ph].

[6] S. Heinemeyer, O. Stal, and G. Weiglein, "Interpreting the LHC Higgs Search Results in the MSSM," Phys.Lett. B710 (2012) 201-206, arXiv:1112.3026 [hep-ph].

[7] O. Buchmueller, R. Cavanaugh, A. De Roeck, M. Dolan, J. Ellis, et al., "Higgs and Supersymmetry," Eur.Phys.J. C72 (2012) 2020, arXiv:1112.3564 [hep-ph].

[8] P. Draper, P. Meade, M. Reece, and D. Shih, "Implications of a $125 \mathrm{GeV}$ Higgs for the MSSM and Low-Scale SUSY Breaking,” Phys.Rev. D85 (2012) 095007, arXiv:1112.3068 [hep-ph].

[9] J. Cao, Z. Heng, D. Li, and J. M. Yang, "Current experimental constraints on the lightest Higgs boson mass in the constrained MSSM,” Phys.Lett. B710 (2012) 665-670, arXiv:1112.4391 [hep-ph].

[10] L. J. Hall, D. Pinner, and J. T. Ruderman, “A Natural SUSY Higgs Near 126 GeV,” JHEP 1204 (2012) 131, arXiv:1112.2703 [hep-ph].

[11] J. Ellis and K. A. Olive, "Revisiting the Higgs Mass and Dark Matter in the CMSSM," Eur.Phys.J. C72 (2012) 2005, arXiv:1202.3262 [hep-ph].

[12] H. Baer, V. Barger, and A. Mustafayev, "Neutralino dark matter in mSUGRA/CMSSM with a 125 GeV light Higgs scalar," JHEP 1205 (2012) 091, arXiv:1202.4038 [hep-ph].

[13] L. Maiani, A. Polosa, and V. Riquer, "Probing Minimal Supersymmetry at the LHC with the Higgs Boson Masses," New J.Phys. 14 (2012) 073029, arXiv:1202.5998 [hep-ph].

[14] T. Cheng, J. Li, T. Li, D. V. Nanopoulos, and C. Tong, "Electroweak Supersymmetry around the Electroweak Scale," arXiv:1202.6088 [hep-ph]. 
[15] J.-J. Cao, Z.-X. Heng, J. M. Yang, Y.-M. Zhang, and J.-Y. Zhu, “A SM-like Higgs near 125 GeV in low energy SUSY: a comparative study for MSSM and NMSSM," JHEP 1203 (2012) 086, arXiv:1202.5821 [hep-ph].

[16] F. Brummer, S. Kraml, and S. Kulkarni, “Anatomy of maximal stop mixing in the MSSM," JHEP 1208 (2012) 089, arXiv:1204.5977 [hep-ph].

[17] C. Balazs, A. Buckley, D. Carter, B. Farmer, and M. White, "Should we still believe in constrained supersymmetry?," arXiv:1205.1568 [hep-ph].

[18] J. L. Feng and D. Sanford, "A Natural $125 \mathrm{GeV}$ Higgs Boson in the MSSM from Focus Point Supersymmetry with A-Terms," Phys.Rev. D86 (2012) 055015, arXiv:1205.2372 [hep-ph].

[19] D. Ghosh, M. Guchait, S. Raychaudhuri, and D. Sengupta, "How Constrained is the cMSSM?," Phys.Rev. D86 (2012) 055007, arXiv:1205.2283 [hep-ph].

[20] A. Fowlie, M. Kazana, K. Kowalska, S. Munir, L. Roszkowski, et al., "The CMSSM Favoring New Territories: The Impact of New LHC Limits and a 125 GeV Higgs,” Phys.Rev. D86 (2012) 075010, arXiv: 1206. 0264 [hep-ph].

[21] P. Athron, S. King, D. Miller, S. Moretti, and R. Nevzorov, "Constrained Exceptional Supersymmetric Standard Model with a Higgs Near 125 GeV," Phys.Rev. D86 (2012) 095003, arXiv:1206. 5028 [hep-ph].

[22] M. W. Cahill-Rowley, J. L. Hewett, A. Ismail, and T. G. Rizzo, "The Higgs Sector and Fine-Tuning in the pMSSM,” Phys.Rev. D86 (2012) 075015, arXiv:1206.5800 [hep-ph].

[23] S. Akula, P. Nath, and G. Peim, "Implications of the Higgs Boson Discovery for mSUGRA," Phys.Lett. B717 (2012) 188-192, arXiv:1207.1839 [hep-ph].

[24] J. Cao, Z. Heng, J. M. Yang, and J. Zhu, "Status of low energy SUSY models confronted with the LHC 125 GeV Higgs data," JHEP 1210 (2012) 079, arXiv:1207.3698 [hep-ph].

[25] A. Arbey, M. Battaglia, A. Djouadi, and F. Mahmoudi, "The Higgs sector of the phenomenological MSSM in the light of the Higgs boson discovery," JHEP 1209 (2012) 107, arXiv:1207.1348 [hep-ph].

[26] P. Nath, "SUGRA Grand Unification, LHC and Dark Matter," arXiv:1207.5501 [hep-ph].

[27] J. Ellis, F. Luo, K. A. Olive, and P. Sandick, “The Higgs Mass beyond the CMSSM," arXiv:1212.4476 [hep-ph].

[28] M. Chakraborti, U. Chattopadhyay, and R. M. Godbole, "Implication of Higgs at $125 \mathrm{GeV}$ within Stochastic Superspace Framework," arXiv:1211.1549 [hep-ph].

[29] A. Arbey, M. Battaglia, A. Djouadi, and F. Mahmoudi, "An update on the constraints on the phenomenological MSSM from the new LHC Higgs results," Phys.Lett. B720 (2013) 153-160, arXiv:1211.4004 [hep-ph].

[30] A. Chakraborty, B. Das, J. L. Diaz-Cruz, D. K. Ghosh, S. Moretti, et al., "The $125 \mathrm{GeV}$ Higgs signal at the LHC in the CP Violating MSSM," arXiv:1301.2745 [hep-ph].

[31] A. Dighe, D. Ghosh, K. M. Patel, and S. Raychaudhuri, "Testing Times for Supersymmetry: Looking Under the Lamp Post," arXiv:1303.0721 [hep-ph].

[32] A. Arbey, A. Deandrea, F. Mahmoudi, and A. Tarhini, "Anomaly mediated supersymmetric models and Higgs data from the LHC," Phys.Rev. D87 (2013) 115020, arXiv:1304.0381 [hep-ph].

[33] G. Belanger, G. D. La Rochelle, B. Dumont, R. M. Godbole, S. Kraml, et al., "LHC constraints on light neutralino dark matter in the MSSM," arXiv:1308.3735 [hep-ph].

[34] A. Djouadi, L. Maiani, G. Moreau, A. Polosa, J. Quevillon, et al., "The post-Higgs MSSM scenario: Habemus MSSM?," arXiv:1307.5205 [hep-ph]. 
[35] R. M. Chatterjee, M. Guchait, and D. Sengupta, "Probing Supersymmetry using Event Shape variables at 8 TeV LHC," Phys.Rev. D86 (2012) 075014, arXiv:1206.5770 [hep-ph].

[36] K. Howe and P. Saraswat, "Excess Higgs Production in Neutralino Decays," JHEP 1210 (2012) 065, arXiv:1208.1542 [hep-ph].

[37] A. Arbey, M. Battaglia, and F. Mahmoudi, "Higgs Production in Neutralino Decays in the MSSM - The LHC and a Future e+e-Collider," arXiv:1212.6865 [hep-ph].

[38] H. Baer, V. Barger, A. Lessa, and X. Tata, "Discovery potential for SUSY at a high luminosity upgrade of LHC14," Phys.Rev. D86 (2012) 117701, arXiv:1207.4846 [hep-ph].

[39] "Search for supersymmetry with the razor variables at cms," Tech. Rep. CMS-PAS-SUS-12-005, CERN, Geneva, July, 2012.

[40] "Search for squarks and gluinos with the atlas detector using final states with jets and missing transverse momentum and $5.8 \mathrm{fb}^{-1}$ of $\sqrt{s}=8$ tev proton-proton collision data," Tech. Rep. ATLAS-CONF-2012-109, CERN, Geneva, Aug, 2012.

[41] E. Cremmer, B. Julia, J. Scherk, P. van Nieuwenhuizen, S. Ferrara, and L. Girardello, "Super-higgs effect in supergravity with general scalar interactions," Physics Letters B 79 no. 3, (1978) 231 - 234. http://www.sciencedirect.com/science/article/pii/0370269378902307.

[42] E. Cremmer, B. Julia, J. Scherk, S. Ferrara, L. Girardello, and P. van Nieuwenhuizen, "Spontaneous symmetry breaking and higgs effect in supergravity without cosmological constant," Nuclear Physics B 147 no. 12, (1979) 105 - 131. http://www. sciencedirect.com/science/article/pii/0550321379904176.

[43] A. H. Chamseddine, R. Arnowitt, and P. Nath, "Locally supersymmetric grand unification," Phys. Rev. Lett. 49 (Oct, 1982) 970-974. http://link.aps.org/doi/10.1103/PhysRevLett.49.970.

[44] L. Hall, J. Lykken, and S. Weinberg, "Supergravity as the messenger of supersymmetry breaking," Phys. Rev. D 27 (May, 1983) 2359-2378. http://link.aps.org/doi/10.1103/PhysRevD . 27.2359.

[45] P. Nath, R. Arnowitt, and A. Chamseddine, "Gauge hierarchy in supergravity $\{$ GUTS $\}$," Nuclear Physics B 227 no. 1 , (1983) $121-133$. http://www.sciencedirect.com/science/article/pii/0550321383901451.

[46] N. Ohta, "Grand unified theories based on local supersymmetry," Progress of Theoretical Physics 70 no. 2, (1983) 542-549, http://ptp.oxfordjournals.org/content/70/2/542.full.pdf+html.

[47] ATLAS Collaboration Collaboration, G. Aad et al., "Search for new phenomena in final states with large jet multiplicities and missing transverse momentum at sqrt(s) $=8 \mathrm{TeV}$ proton-proton collisions using the ATLAS experiment," arXiv:1308.1841 [hep-ex].

[48] "Search for squarks and gluinos with the ATLAS detector in final states with jets and missing transverse momentum and $20.3 \mathrm{fb}^{-1}$ of $\sqrt{s}=8 \mathrm{TeV}$ proton-proton collision data," Tech. Rep. ATLAS-CONF-2013-047, CERN, Geneva, May, 2013.

[49] "Search for strong production of supersymmetric particles in final states with missing transverse momentum and at least three b-jets using $20.1 \mathrm{fb} 1$ of pp collisions at sqrt(s) $=8$ tev with the atlas detector.," Tech. Rep. ATLAS-CONF-2013-061, CERN, Geneva, Jun, 2013.

[50] A. G. Cohen, D. Kaplan, and A. Nelson, "The More minimal supersymmetric standard model," Phys.Lett. B388 (1996) 588-598, arXiv: hep-ph/9607394 [hep-ph].

[51] J. Berger, J. Hubisz, and M. Perelstein, “A Fermionic Top Partner: Naturalness and the LHC," JHEP 1207 (2012) 016, arXiv:1205.0013 [hep-ph].

[52] J. Cao, C. Han, L. Wu, J. M. Yang, and Y. Zhang, "Probing Natural SUSY from Stop Pair Production at the LHC," arXiv:1206.3865 [hep-ph]. 
[53] L. Randall and M. Reece, "Single-Scale Natural SUSY," arXiv:1206.6540 [hep-ph].

[54] J. R. Espinosa, C. Grojean, V. Sanz, and M. Trott, "NSUSY fits," JHEP 1212 (2012) 077, arXiv: 1207.7355 [hep-ph].

[55] H. Baer, V. Barger, P. Huang, D. Mickelson, A. Mustafayev, et al., "Post-LHC7 fine-tuning in the mSUGRA/CMSSM model with a 125 GeV Higgs boson,” Phys.Rev. D87 no. 3, (2013) 035017, arXiv:1210.3019 [hep-ph].

[56] M. Papucci, J. T. Ruderman, and A. Weiler, “Natural SUSY Endures,” JHEP 1209 (2012) 035, arXiv:1110.6926 [hep-ph].

[57] N. Desai and B. Mukhopadhyaya, "Constraints on supersymmetry with light third family from LHC data," JHEP 1205 (2012) 057, arXiv:1111.2830 [hep-ph].

[58] B. He, T. Li, and Q. Shafi, "Impact of LHC Searches on NLSP Top Squark and Gluino Mass,” JHEP 1205 (2012) 148, arXiv:1112.4461 [hep-ph].

[59] M. Drees, M. Hanussek, and J. S. Kim, "Light Stop Searches at the LHC with Monojet Events," Phys. Rev. D86 (2012) 035024, arXiv:1201.5714 [hep-ph].

[60] T. Plehn, M. Spannowsky, and M. Takeuchi, “Stop searches in 2012," JHEP 1208 (2012) 091, arXiv:1205.2696 [hep-ph].

[61] Z. Han, A. Katz, D. Krohn, and M. Reece, “(Light) Stop Signs,” JHEP 1208 (2012) 083, arXiv: 1205.5808 [hep-ph].

[62] V. Barger, P. Huang, M. Ishida, and W.-Y. Keung, "Scalar-Top Masses from SUSY Loops with 125 GeV mh and Precise Mw," arXiv:1206.1777 [hep-ph].

[63] A. Choudhury and A. Datta, "New limits on top squark NLSP from LHC $4.7 f b^{-1}$ data," Mod.Phys.Lett. A27 (2012) 1250188, arXiv:1207.1846 [hep-ph].

[64] C.-Y. Chen, A. Freitas, T. Han, and K. S. Lee, "New Physics from the Top at the LHC," arXiv: 1207.4794 [hep-ph].

[65] S. Bornhauser, M. Drees, S. Grab, and J. Kim, "Light Stop Searches at the LHC in Events with two b-Jets and Missing Energy," Phys.Rev. D83 (2011) 035008, arXiv:1011.5508 [hep-ph].

[66] S. Kraml and A. Raklev, "Same-sign top quarks as signature of light stops at the LHC," Phys.Rev. D73 (2006) 075002, arXiv:hep-ph/0512284 [hep-ph].

[67] Z.-H. Yu, X.-J. Bi, Q.-S. Yan, and P.-F. Yin, "Detecting light stop pairs in coannihilation scenarios at the LHC," arXiv:1211.2997 [hep-ph].

[68] M. A. Ajaib, T. Li, and Q. Shafi, "Stop-Neutralino Coannihilation in the Light of LHC," Phys.Rev. D85 (2012) 055021, arXiv:1111.4467 [hep-ph].

[69] K. Ghosh, K. Huitu, J. Laamanen, L. Leinonen, K. Huitu, et al., "Top jets as a probe of degenerate stop-NLSP LSP scenario in the framework of cMSSM," arXiv:1207.2429 [hep-ph].

[70] B. Dutta, T. Kamon, N. Kolev, K. Sinha, and K. Wang, "Searching for Top Squarks at the LHC in Fully Hadronic Final State," Phys.Rev. D86 (2012) 075004, arXiv:1207.1873 [hep-ph].

[71] D. S. Alves, M. R. Buckley, P. J. Fox, J. D. Lykken, and C.-T. Yu, "Stops and MET: The Shape of Things to Come," arXiv:1205.5805 [hep-ph].

[72] D. Berenstein, T. Liu, and E. Perkins, "Multiple b-jets reveal natural SUSY and the 125 GeV Higgs," arXiv:1211.4288 [hep-ph].

[73] D. Ghosh and D. Sengupta, "Searching the sbottom in the four lepton channel at the LHC," Eur.Phys.J. C73 (2013) 2342, arXiv:1209.4310 [hep-ph]. 
[74] A. Chakraborty, D. K. Ghosh, D. Ghosh, and D. Sengupta, "Stop and sbottom search using dileptonic $M_{T 2}$ variable and boosted top technique at the LHC," arXiv:1303.5776 [hep-ph].

[75] D. Ghosh, "Boosted di-boson from a mixed heavy stop," arXiv:1308.0320 [hep-ph].

[76] C. Han, K.-i. Hikasa, L. Wu, J. M. Yang, and Y. Zhang, "Current experimental bounds on stop mass in natural SUSY," arXiv:1308.5307 [hep-ph].

[77] O. Buchmueller and J. Marrouche, "Universal mass limits on gluino and third-generation squarks in the context of Natural-like SUSY spectra," arXiv:1304.2185 [hep-ph].

[78] G. D. Kribs, A. Martin, and A. Menon, "Natural Supersymmetry and Implications for Higgs physics," Phys.Rev. D88 (2013) 035025, arXiv: 1305.1313 [hep-ph].

[79] K. Kowalska and E. M. Sessolo, "Natural MSSM after the LHC 8 TeV run," Phys.Rev. D88 (2013) 075001, arXiv:1307.5790 [hep-ph].

[80] D. E. Kaplan, K. Rehermann, and D. Stolarski, "Searching for Direct Stop Production in Hadronic Top Data at the LHC," JHEP 1207 (2012) 119, arXiv:1205.5816 [hep-ph].

[81] T. Plehn, M. Spannowsky, and M. Takeuchi, "How to Improve Top Tagging," Phys.Rev. D85 (2012) 034029, arXiv:1111.5034 [hep-ph].

[82] "Search for direct top squark pair production in final states with one isolated lepton, jets, and missing transverse momentum in sqrt(s) $=8$ tev pp collisions using 13.0 ifb of atlas data," Tech. Rep. ATLAS-CONF-2012-166, CERN, Geneva, Dec, 2012.

[83] "Search for direct stop production in events with missing transverse momentum and two b-jets using 12.8 $f b-1$ of pp collisions at sqrts $=8$ tev with the atlas detector," Tech. Rep. ATLAS-CONF-2013-001, CERN, Geneva, Jan, 2013.

[84] "Search for a supersymmetric top-quark partner in final states with two leptons in sqrt(s) $=8$ tev pp collisions using 13 ifb of atlas data," Tech. Rep. ATLAS-CONF-2012-167, CERN, Geneva, Dec, 2012.

[85] "Search for direct top squark pair production in events with a single isolated lepton, jets and missing transverse energy at sqrt(s) $=8$ tev,".

[86] K.-i. Hikasa and M. Kobayashi, "Light scalar top quark at $e^{+} e^{-}$colliders," Phys. Rev. D 36 (Aug, 1987) 724-732. http://link.aps.org/doi/10.1103/PhysRevD.36.724.

[87] M. Muhlleitner and E. Popenda, "Light Stop Decay in the MSSM with Minimal Flavour Violation," JHEP 1104 (2011) 095, arXiv:1102.5712 [hep-ph].

[88] C. Boehm, A. Djouadi, and Y. Mambrini, "Decays of the lightest top squark," Phys.Rev. D61 (2000) 095006, arXiv:hep-ph/9907428 [hep-ph].

[89] A. Djouadi, M. Guchait, and Y. Mambrini, "Scalar top quarks at the run II of the Tevatron in the high tan beta regime," Phys.Rev. D64 (2001) 095014, arXiv: hep-ph/0105108 [hep-ph].

[90] S. P. Das, A. Datta, and M. Guchait, "Four-body decay of the stop squark at the upgraded Tevatron," Phys.Rev. D65 (2002) 095006, arXiv: hep-ph/0112182 [hep-ph].

[91] M. Carena, A. Freitas, and C. Wagner, "Light Stop Searches at the LHC in Events with One Hard Photon or Jet and Missing Energy," JHEP 0810 (2008) 109, arXiv:0808.2298 [hep-ph].

[92] G. Belanger, M. Heikinheimo, and V. Sanz, "Model-Independent Bounds on Squarks from Monophoton Searches," JHEP 1208 (2012) 151, arXiv:1205.1463 [hep-ph].

[93] "Search for new phenomena in monojet plus missing transverse momentum final states using $1 \mathrm{fb}-1$ of pp collisions at sqrts=7 tev with the atlas detector," Tech. Rep. ATLAS-CONF-2011-096, CERN, Geneva, Jul, 2011. 
[94] CMS Collaboration Collaboration, S. Chatrchyan et al., "Search for dark matter and large extra dimensions in monojet events in $p p$ collisions at $\sqrt{s}=7 \mathrm{TeV}$," JHEP 1209 (2012) 094, arXiv:1206.5663 [hep-ex].

[95] MSSM Working Group Collaboration, A. Djouadi et al., "The Minimal supersymmetric standard model: Group summary report," arXiv:hep-ph/9901246 [hep-ph].

[96] A. Djouadi, J.-L. Kneur, and G. Moultaka, "SuSpect: A Fortran code for the supersymmetric and Higgs particle spectrum in the MSSM," Comput.Phys.Commun. 176 (2007) 426-455, arXiv : hep-ph/0211331 [hep-ph].

[97] F. Mahmoudi, "SuperIso: A Program for calculating the isospin asymmetry of B in the MSSM," Comput.Phys.Commun. 178 (2008) 745-754, arXiv:0710.2067 [hep-ph].

[98] Heavy Flavor Averaging Group Collaboration, D. Asner et al., "Averages of b-hadron, c-hadron, and $\tau$-lepton Properties," arXiv: 1010.1589 [hep-ex].

[99] Heavy Flavor Averaging Group Collaboration, Y. Amhis et al., "Averages of B-Hadron, C-Hadron, and tau-lepton properties as of early 2012," arXiv:1207.1158 [hep-ex].

[100] A. J. Buras and J. Girrbach, "Towards the Identification of New Physics through Quark Flavour Violating Processes," arXiv:1306.3775 [hep-ph].

[101] J. R. Ellis, S. Heinemeyer, K. Olive, A. Weber, and G. Weiglein, "The Supersymmetric Parameter Space in Light of $B^{-}$physics Observables and Electroweak Precision Data," JHEP 0708 (2007) 083, arXiv:0706.0652 [hep-ph].

[102] LHCb collaboration Collaboration, R. Aaij et al., "Measurement of the $B_{s}^{0} \rightarrow \mu^{+} \mu^{-}$branching fraction and search for $B^{0} \rightarrow \mu^{+} \mu^{-}$decays at the LHCb experiment," arXiv:1307.5024 [hep-ex].

[103] CMS Collaboration Collaboration, S. Chatrchyan et al., "Measurement of the $B_{s}^{0} \rightarrow \mu^{+} \mu^{-}$branching fraction and search for $B^{0} \rightarrow \mu^{+} \mu^{-}$with the CMS Experiment," arXiv:1307.5025 [hep-ex].

[104] T. Sjostrand, S. Mrenna, and P. Z. Skands, "PYTHIA 6.4 Physics and Manual," JHEP 0605 (2006) 026, arXiv:hep-ph/0603175 [hep-ph].

[105] M. L. Mangano, M. Moretti, F. Piccinini, R. Pittau, and A. D. Polosa, "ALPGEN, a generator for hard multiparton processes in hadronic collisions," JHEP 0307 (2003) 001, arXiv: hep-ph/0206293 [hep-ph].

[106] M. Cacciari, G. P. Salam, and G. Soyez, “FastJet User Manual,” Eur.Phys.J. C72 (2012) 1896, arXiv:1111.6097 [hep-ph].

[107] M. Cacciari, G. P. Salam, and G. Soyez, “The Anti-k(t) jet clustering algorithm," JHEP 0804 (2008) 063, arXiv:0802.1189 [hep-ph].

[108] S. Hoeche, F. Krauss, N. Lavesson, L. Lonnblad, M. Mangano, et al., "Matching parton showers and matrix elements," arXiv:hep-ph/0602031 [hep-ph].

[109] CTEQ Collaboration, H. Lai et al., "Global QCD analysis of parton structure of the nucleon: CTEQ5 parton distributions," Eur.Phys.J. C12 (2000) 375-392, arXiv: hep-ph/9903282 [hep-ph].

[110] D. Bourilkov, R. C. Group, and M. R. Whalley, "LHAPDF: PDF use from the Tevatron to the LHC," arXiv:hep-ph/0605240 [hep-ph].

[111] L. Randall and D. Tucker-Smith, "Dijet Searches for Supersymmetry at the LHC," Phys.Rev.Lett. 101 (2008) 221803, arXiv:0806.1049 [hep-ph].

[112] A. Barr, C. Lester, and P. Stephens, “m(T2): The Truth behind the glamour," J.Phys. G29 (2003) 2343-2363, arXiv:hep-ph/0304226 [hep-ph].

[113] C. Lester and D. Summers, "Measuring masses of semiinvisibly decaying particles pair produced at hadron colliders," Phys.Lett. B463 (1999) 99-103, arXiv: hep-ph/9906349 [hep-ph]. 
[114] A. J. Barr and C. Gwenlan, "The Race for supersymmetry: Using m(T2) for discovery,” Phys.Rev. D80 (2009) 074007, arXiv:0907.2713 [hep-ph].

[115] "Measurement of associated charm production in $\mathrm{w}$ final states at sqrt(s) $=7$ tev," Tech. Rep. CMS-PAS-SMP-12-002, CERN, Geneva, 2013.

[116] "Search for pair-produced top squarks decaying into a charm quark and the lightest neutralinos with $20.3 \mathrm{fb}^{-1}$ of $p p$ collisions at $\sqrt{s}=8$ tev with the atlas detector at the lhc," Tech. Rep. ATLAS-CONF-2013-068, CERN, Geneva, Jul, 2013.

[117] W. Beenakker, R. Hopker, and M. Spira, "PROSPINO: A Program for the production of supersymmetric particles in next-to-leading order QCD," arXiv:hep-ph/9611232 [hep-ph].

[118] N. Kidonakis, “Top Quark Theoretical Cross Sections and pT and Rapidity Distributions,” arXiv:1109. 3231 [hep-ph].

[119] C. Berger, Z. Bern, L. J. Dixon, F. Febres Cordero, D. Forde, et al., "Precise Predictions for W + 4 Jet Production at the Large Hadron Collider," Phys.Rev.Lett. 106 (2011) 092001, arXiv:1009. 2338 [hep-ph].

[120] H. Ita, Z. Bern, L. Dixon, F. Febres Cordero, D. Kosower, et al., "Precise Predictions for Z + 4 Jets at Hadron Colliders," Phys.Rev. D85 (2012) 031501, arXiv:1108.2229 [hep-ph].

[121] Planck Collaboration Collaboration, P. Ade et al., "Planck 2013 results. XVI. Cosmological parameters," arXiv:1303.5076 [astro-ph.C0].

[122] G. Belanger, F. Boudjema, A. Pukhov, and A. Semenov, "micrOMEGAs3.1 : a program for calculating dark matter observables," arXiv:1305.0237 [hep-ph]. 